ta dominica sacratissimę quadragesimę consecrari magno misterio et alicui ex primoribus christianorum regi vel principi dicari et mitti solitam, hoc anno dilecto filio nobili viro Federico duci Saxonie, utpote suorum clarissimorum progenitorum more de nobis et sancta apostolica sede bene merito, utque posthac magis mereri possit, dicaverimus, ex dilecto filio Carolo Militz nuncio, cubiculario secreto et familiari nostro, et quedam, que nos sedisque praedictę dignitatem auctoritatemque respiciunt, devotio tua plenius intelliget. Scientes insuper et merito quidem, quanta sit devotionis tuę apud eundem ducem gratia, quantive ille salubre ac prudens consilium tuum faciet, devotionem ipsam tuam hortamur in Domino ac paterne requirimus, ut pro sua debita erga nos eandemque sedem devotione et observantia recte considerans. quanto decore, quove munere eundem ducem dignum duxerimus, considerans etiam, quam detestabilis sit unius Sathane filii fratris Martini Lotter nimia temeritas, que etiam et notissimam ${ }^{2}$ heresim sapit et tanti ducis clarum nomen, claram etiam suorum progenitorum famam denigrare potest, eodem Carolo nuntio nostro audito ea eidem duci suo sano consilio persuadere velit, per que nostrę et dictę sedis dignitati et eiusdem ducis decori recte consulatur et dicti Martini temeritas comprimatur et error, heu nimium gravis, qui in populo plerunque nimium credulo ita seminatur, te uno potissimum rem iuvante teque bono consultore tollatur. In quo devotio eadem tua Deo salvatori nostro, cuius causa agitur, rem acceptam et nobis, qui nil magis quam zizaniam loliumque huiusmodi ex agro Domini $i^{3}$ extirpari posse studemus, gratissimam faciet. Pro qua in suis etiam piis votis et desideriis devotio eadem tua nos sedemque praedictam magis sibi propicios inveniet atque benignos, prout ex eodem Carolo a nobis plene instructo eadem devotio plenius intelliget. Datum Civitatis ${ }^{4}$ veteris Viterbiensis diocesis, sub annulo piscatoris, die XXIIIIto octobris MDXVIII, pontificatus nostri anno sexto.

+ Evangelista subscripsit ${ }^{6}$.

' tissimam von einer zweiten Hand auf Rasur, dabei die letzte Silbe hochgestellt, vgl, bei Anm. 73.

Vgl. Mt 13, 25-30

${ }^{4}$-is veteris bis pontificatus von der zweiten Hand.

${ }^{5}$ So Or., wie seit 1455 in Breven üblich, vgl. Th. FRENZ, Das Eindringen humanistischer Schriftformen in die Urkunden und Akten der päpstlichen Kurie im 15. Jahrhundert, ArchDipl 20 (1974) S. 425.

${ }^{6}$ Eigenhändige Unterschrift von Evangelista Tarasconus (Tarasconio), seit dem 10. März 1511 apostolischer Sekretär (Secretarius apostolicus), bei seinem Tod im September 1531 Geheimsekretär Clemens' VII., HOFMANN, Forschungen 2 (wie Anm. 57), S. 121, 124, FRENZ, Kanzlei (wie Anm. 118), S. 320 Nr. 631.

\section{Emblematische Viten von Jesuitenheiligen im 17./18. Jahrhundert}

von Éva Knapp und Gábor Tüskés

Die Forschung zur Jesuitenemblematik erlebt in den letzten zwei Jahrzehnten einen großen Aufschwung. ${ }^{.}$Ein gut definierbares Gebiet innerhalb dieses Forschungsfeldes bilden die Emblemserien, die mit Leben, Wundern und Tugenden der Jesuitenheiligen bzw. der ihrer heiligen Lebensführung nach berühmten Jesuiten im Zusammenhang stehen. Auf diese Gattung der Gebrauchsliteratur hat bereits Praz aufmerksam gemacht, als er davor warnte, Publikationen, die illustrierte Szenen mit einigen Zeilen Erklärungen zusammen aus dem Leben der Heiligen enthalten, willkürlich unter die Emblembücher einzureihen. ${ }^{2}$ Wie es inzwischen nachgewiesen wurde, erschienen seit den ersten zwei Jahrzehnten des 17. Jahrhunderts, neben der traditionellen Gattung der illustrierten Heiligenviten und parallel zu den Kanonisationsbestrebungen der Jesuiten, in immer größerer Zahl Emblemserien über die tatsächlichen und potentiellen Heiligen des Jesuitenordens. Diese Serien stellen einen bedeutenden Teil der hervorragenden Persönlichkeiten gewidmeten Drucke innerhalb der Gattung der heroischen Emblematik. In den emblematischen Heiligenviten, die einen Erzählzusammenhang bilden, wird das Lebensganze in signifikante Stationen aufgelöst, an denen das Beispielhafte dieses Lebens in einzelnen Ereignissen entweder als Eingriffe der göttlichen Providenz oder als persönliche Erfüllung vorgegebener Verhaltensmuster sichtbar gemacht, verbal bestätigt und mit der Aufforderung zur Imitatio verbunden wird.

G. Richard DiMLER, Jesuitische Emblembücher. Zum Forschungsstand, in: Sinnbild - Bildsinn. Emblembücher der Stadtbibliothek Trier. Katalogbuch zur Ausstellung, Red. Michael Schunck, Trier 1991, S. 169-174.

Mario PraZ, Studies in Seventeenth-Century Imagery. Roma 1964, S. 326. Zu den Emblemprogrammen der Heiligen in der kirchlichen Kunst vgl. Reallexikon der deutschen Kunstgeschichte, Bd. V, Stuttgart 1967, Sp. 193-195, Abb, 40 a-b; Cornelia KeMP, Angewandte Emblematik in süddeutschen Barockkirchen, München - Berlin 1981. S, 46-48, 53-75. 
Zum einen bieten die emblematischen Viten der Jesuitenheiligen die Möglichkeit, das Auftauchen eines Themas, seine Verbreitung und sein Absterben in der Emblemtradition zu verfolgen. Zum anderen liefert eine solche Untersuchung Kenntnisse über den Spezialisierungsprozeß, den die Jesuitenemblematik hinsichtlich ihrer Stoffe und Anlässe durchlief. Darüber hinaus wirft sie ein neues Licht auf bisher ungeklärte Fragen der Assimilation des humanistischen Emblems durch die Jesuiten sowie auf ihre spezielle Arbeitsmethode. ${ }^{3}$

\section{Theoretischer Hintergrund und Entstehung der Gattung}

Die Ausdruckskraft des Emblems wird außer durch Begabung und Können seines Schöpfers grundlegend von der Qualität der darin versteckten intellektuellen Erfindung bestimmt. Dadurch konnte es über seine Funktion als Anziehungspunkt für die Aufmerksamkeit hinaus zur Quelle neuen Wissens werden. Die Exercitia Spiritualia des Ignatius von Loyola liefen ja ebenfalls auf eine Erfahrung hinaus, die im Empfänger wieder aufgebaut, vertieft und erlebt werden sollte. Ignatius jedoch lehnte in den Exercitien die Suche nach einem Gleichgewicht zwischen intellektuellem Wissen und Erleben $a^{4}$ : Um einer erlebten religiösen Erfahrung teilzuwerden, bevorzugte er zuungunsten der ,abundantia scientiae“ den bewußten Gebrauch der Sinne.

Die Verwandtschaft zwischen den Eigentümlichkeiten des humanistischen Emblems und den Exerzitien des Ignatius haben die Jesuiten bereits am Ende des 16. Jahrhunderts entdeckt. Die Meditationen von Jeronimo Nadal und Jacobus Pontanus befolgten nicht nur die Vorschriften von Ignatius, sondern verrieten zugleich eine starke Affinität zur Emblematik. ${ }^{5}$ Schon bald nach ihrem Wirken erschienen die ersten illustrierten Ausgaben der ignazia-

G. Richard DimLer, The Imago Primi Saeculi: Jesuit Emblems and the Secular Tradition. in: Tought 56 (1981), S. 433-448. Vgl. Dean-Marc Chatelain, Livres d'emblèmes et de devises. Une anthologie (1531-1735), s. L. 1993; Heribert BREIDENBACH, Der Emblematiker Jeremias Drexel S. J. (1581 bis 1638). Mit einer Einführung in die Jesuitenemblematik und einer Bibliographie der Jesuitenemblembücher. Diss. Phil. University of lllinois, Urbana 1970. S. 79-89.

${ }^{4}$ In der Einführung der Exercitia: „non enim abundantia scientiae, sed sensus et gustus rerum interior desiderium animae explere solet." Sancti Ignatii de Loyola Exercitia Spiritualia. Textus antiquissimi, Ed. I. Calveras - C. de Dalmases, Roma 1969, S. 142-143.

'Jeronimo NADAL, Evangelicae historiae imagines, Antwerpen 1593; DERS., Adnotationes et meditationes, Antwerpen 1594-1595; Jacobus Pontanus (Spanmüller), Floridorum libri octo. Augustae Vindelicorum 1595 nischen Exerzitien, ${ }^{6}$ darunter eine Edition aus dem 17. Jahrhundert, die mit den Bildern eines ursprünglich nicht für die Illustrierung der Exerzitien gedachten jesuitischen Emblembuches ausgestattet wurde. ${ }^{?}$

Gleichzeitig damit begannen die Jesuiten, Formenwelt und Inventar der Emblematik gemäß den Ansprüchen des Ordens herauszuarbeiten. Im Laufe dieses längeren Prozesses entwickelten sie eine eigene Theorie und fanden deren Wurzeln in den Vorstellungen der humanistischen Emblematik des 16. Jahrhunderts, insbesondere in den italienischen Symbol-(Impresen)Theorien. Diese Ausdrucksform entsprach den jesuitischen Zielen insofern besser als die Emblematik, als man durch sie statt abstrakter Ideen und komplizierter Gedanken konkrete, jene ,abundantia scientiae“ entbehrende Inhalte zum Ausdruck bringen konnte. Die Impresentheorie wurde in Italien gerade zu der Zeit in weiteren Kreisen bekannt und beliebt, als sich der neugegründete Orden der Jesuiten zu einer starken Organisation entwickelte. ${ }^{8}$

Das Interesse der Jesuiten am Symbol kommt zum ersten Mal, wenngleich nur indirekt, in Roberto Bellarminos imago-Theorie ${ }^{9}$ zum Ausdruck. Für ihn stellt das Bild nicht nur einen mit Hilfe der optischen Wahrnehmung erfahrbaren Gegenstand dar, sondern zugleich eine wirkliche Ähnlichkeit mit den Dingen der Welt, zu der man - wie bei Ignatius - mittels der Sinne Zugang finden kann ${ }^{10}$. Bellarmino führte den Begriff der ,utilitas imaginis“ ein, wonach die imago

1. ein Mittel der Belehrung und Unterhaltung ist;

2. der Pflege und Stärkung der Demut gegenüber Gott und den Heiligen dient;

3. zur Nachahmung des Beispiels anspornt;

4. dem Menschen hilft, die Erinnerung an Christus und die Heiligen wachzuhalten;

5. ein Mittel des Glaubensbekenntnisses ist ${ }^{11}$. 1649

"Ignatius DE LOYOLA, Exercitia spiritualia, Romae 1609; DERS., Esertitii spirituali, Roma 'Ignatius DE Loyola, Exercitia spiritualia, Tyrnaviae 1679 ,

${ }^{8}$ Vgl. Dieter Sulzer, Traktate zur Emblematik, Studien zu einer Geschichte der Emblemtheorien, Hg, von Gerhard Sauder, St. Ingbert 1992.

${ }^{9}$ Roberto Bellarmino, Septimana controversia generalis de Ecclesia triumphante tribus libris explicata. Liber secundus. De reliquiis et imaginibus Sanctorum, in: DERS.. Opera omnia, Ed. Justinus Fèvre, Tom. III, Parisiis 1870, S. 199-266. (Erstausgabe: Ingolstadt 1586).

${ }^{10}$ Ebd., S. 213.

${ }^{11}$ Ebd., S. 228-229. 
Seine Theorie hat dazu beigetragen, daß die Jesuiten aufgrund des utilitasPrinzips in die Reihe der dem Ausdruck religiöser Inhalte dienenden literarischen Formen (himnus, cantata, apophtegmata, litania, exemplum, carmen, elogium heroicum usw.) auch das bildhafte Emblem aufnahmen.

Fast gleichzeitig damit entstand im Orden der Bedarf nach neuen Mitteln, mit denen man wirksamer als mit den bereits bekannten mittelalterlichen Formen der Kultverbreitung für die Verehrung der heiligen Ordensmitglieder werben konnte. Im Zuge der Kanonisationsbestrebungen der Jesuiten, die sich um die Wende vom 16. zum 17. Jahrhundert entfalteten, ist die bildliche Darstellung, die Herausarbeitung der ,,vera imago“ der künftigen Heiligen, zum wichtigen Bestandteil des ,,vita et miracula“-Programms geworden ${ }^{12}$. Eine weitere Form der Kultvermittlung stellten die aus dem ,vita et miracula“Ideenkreis herausgelösten, auch selbständig herausgearbeiteten und durch spezifisch jesuitische Tugenden erweiterten virtutes-Zusammenstellungen dar. Nicht unabhängig von diesen Formen stellten die Jesuiten als dritte die Emblematik in den Dienst ihrer Kanonisationspläne, da sie in ihr ein geeignetes Mittel sahen, den Kult der noch nicht bekannten, mit den Zeichen der Heiligkeit ausgestatteten Personen in weiteren Kreisen bekannt zu machen.

Der erste Emblematiker unter den Jesuiten, Jacobus Pontanus (Spanmüller), löste aus der humanistischen Emblematik die Begriffe der similitudo und delectatio heraus und ordnete sie dem ,utilitas“-Gedankenkreis zu ${ }^{13}$. Antonio Possevino (1593) empfahl das zum Ausdruck des Individuellen besonders geeignete Symbol zur Darstellung von pietas, virtutes, honestas und anima. Diese beziehen sich seiner Ansicht nach auf die Heiligen, und können wirksam dazu beitragen, die neuen Heiligen bekannt zu machen und den Wunsch nach imitatio zu wecken ${ }^{14}$. Horatio Montaldo trat dafür ein, das Symbol von den für versteckte Mitteilungen geeigneten Ausdrucksformen (wie z.B. nota, aenigma, emblema, parabola, apologus und hieroglyphum) zu trennen ${ }^{15}$. Die theoretische Begründung dazu wurde von Nicolas Caussin geliefert ${ }^{16}$. Caus-

${ }^{2}$ Ursula KÖNIG-NORDHOFF, Ignatius von Loyola. Studien zur Entwicklung einer neuen Heiligen-Ikonographie im Rahmen einer Kanonisationskampagne um 1600, Berlin 1982.

13 Jacobus PoNTANus (Spanmüller), Poeticarum institutionum libri III. Editio secunda, Ingolstadii 1597, S. 188-190; DERS., Floridorum (wie Anm. 5), f. 2a-5b.

${ }^{14}$ Antonio Possevino, Bibliotheca selecta de ratione studiorum, Coloniae 1607, S. 479-480.

${ }^{15}$ Horatio MonTaldo, Riposte di Hercole Tasso, Mediolano (1612): Vgl. Claude-Francois MenEstrIER, Philosophia imaginum, Amstelodami - Gedani 1695, S. 22-23. (Erstausgabe: Paris 1682)

${ }^{16}$ Nicolas CAUSsIN, De symbolica Aegyptiorum sapientia, Coloniae 1631, f. 6b-8b. (Erstausgabe: Paris 1618) sin erhob das so getrennte Symbol als das Mittel, das in der Praxis jesuitischer Emblematik und zur Heiligendarstellung am effektivsten einzusetzen sei, zu einer Art Oberkategorie und stellte die geeignete Ähnlichkeit (similitudo idonea), die bereits für Montaldo zu den wichtigsten Elementen des Symbols gehörte, als den Schlüsselbegriff bei der Beschreibung der Funktion des Emblems heraus. Damit ist die „similitudo idonea“ zu einer der wichtigsten Forderungen in der Darstellung der Heiligen geworden. Von da an waren Emblem und Symbol synonyme Begriffe für die Jesuiten, die sich im Gebrauch immer wieder kreuzten.

Alessandro Donati behandelte die Emblematik in erster Linie unter dem Aspekt der Funktion ${ }^{17}$. Die Symbole definiert er als ,figurata epigrammata“, und ihr Sinn bestehe vor allem darin, daß sie ,monent et docent". Als Beispiele dafür zitiert Donati die Ignatius-Symbole des Fammiano Strada, die außer den erwähnten Funktionen auch der Forderung nach Verständlichkeit entsprechen würden. Für Silvestro Pietrasanta war die Würde (dignitas) der Symbole besonders wichtig ${ }^{18}$, die mittels einer verherrlichenden und verehrungsvollen bildlichen und literarischen Darstellung zu erreichen sei.

Neben den theoretischen Überlegungen spielten in der Entstehung der Gattung vor allem die Kanonisationsbestrebungen des Ordens eine wichtige Rolle. Nach dem Tod von Joannes Codurius (August 1541) entwickelte sich der Brauch, daß ein Ordensmitglied in einem kurzem Brief, dem sog. Elogium, über Leben und Wirken des Verstorbenen berichtete ${ }^{19}$. Neben den regelmäßig verfaßten und verbreiteten Elogien achtete man darauf, die Vita der Jesuiten, die durch ihre Tugenden, ihr Leben und Wirken besonders hervorragten, auch in ausführlicherer Form aufzuzeichnen und zu verbreiten ${ }^{20}$.

Neben Elogien enstanden ab und zu auch andere Schriften mit ähnlichem Zweck. So wurde z.B über Stanislaus Kostka außer dem handschriftlichen Elogium noch im Jahre seines Todes eine erste, ebenfalls handschriftliche Fassung seiner Vita verfaßt ${ }^{21}$. Im Jahre 1570 beschrieb man sein Leben in

${ }_{17}^{17}$ Alessandro Donat1, Ars poetica [...] libri tres, Romae 1631, S. 375-388.

${ }^{18}$ Silvestro Pietrasanta, De symbolis heroicis libri IX, Antverpiae 1634, S. 10-13, 352.

${ }^{19}$ Constitutiones Societatis lesu et Epitome Instituti, Romae 1943, S. 134-138.

${ }^{20}$ Elogia virorum illustrium Societatis lesu Cardinalium, episcoporum, martyrum aliorumque sanctitate insignium. Saec. XVII. Universitätsbibliothek Budanest, Ms. Ab 132.

${ }^{21}$ Giulio Fatıo, Stanislai Kostka beata mors et elogium, 1568. MS. Vgl. Carlos SommervoGEL (ed.), Bibliothèque de la Compagnie de Jesus. Première partie: Bibliographie, I-XIII. Bruxelles - Paris - Toulouse 1890-1914. hier: III. Sp. 552: Stanislas WARSZEWICKI, Vita S. Stanislai Kostka, 1568. Ed. Hagiographi Bollandiani, Bruxellis 1895. 
einem carmen, in dem man ihn als ,,selig“ bezeichnete ${ }^{22}$. Zwei Jahre später wurde dann die erste Vita von Ignatius von Loyola veröffentlicht ${ }^{23}$.

Neben den Ordensmitgliedern, die aufgrund ihrer besonderen Tugenden und vorbildlichen Lebensführung bereits in ihrem Leben bekannt geworden und deren Viten bald nach ihrem Tode erschienen sind, stellte man über die gleichermaßen tugendhaften, jedoch weniger bekannten Mitglieder des Ordens ebenfalls handschriftliche Aufzeichnungen zusammen. Diese wurden dann nach Rom geschickt, wo sie nach ihrer Prüfung durch die Ordensleitung für die Bekanntmachung dieser Personen in breiteren Kreisen verwendet wurden ${ }^{24}$.

Für die emblematischen Lebensbeschreibungen bildete die als authentisch anerkannte Vita-Zusammenstellung die Hauptquelle. Die enge Beziehung zwischen den beiden Gattungen und der bereits feste Gebrauch sollen hier am Beispiel des Joannes Franciscus Regis aufgezeigt werden. Nach Regis' Tod (31. Dezember 1640) sind mehrere Ordensviten (Claudius La Brouë, 1650; Antonius Bonnet, Toulouse 1692) entstanden. Als dann seine Kanonisation in Erwägung gezogen wurde, hat General Tamburini Guillelmus Daubenton mit der Anfertigung der authentischen Vita beauftragt. Diese erschien 1716 in Paris in französischer Sprache ${ }^{25}$. Die Seligsprechung im Jahre 1726 und die 1737 erfolgte Heiligsprechung beruhten zum Teil auf Daubentons Vita, die sich als wirksames Mittel in der Verbreitung der Verehrung erwies. Der Vergleich der emblematischen Lebensbeschreibungen über Regis mit der Vita des Daubenton zeigt, daß in ihnen nur solche Motive vorkommen, die auch in der Vita zu finden sind ${ }^{26}$. Nach einer Bemerkung der als Descriptio sacri apparatus betitelten emblematischen Lebensbeschreibung diente Daubentons Vita als Textquelle für die Embleme ${ }^{27}$.

\footnotetext{
Pedro Ribadeneyra, Vita Ignatii Loiolae, Neapoli 1572.

${ }^{24}$ Vgl. Joannes VINCARTIUS, Sacrarum heroidum epistolae, Tornaci 1640; Imago Primi Saeculi, Antverpiae 1640; Ignatius QueRCK, Acta S. Ignatii de Lojola, Viennae 1698.

25 Guillelmus DauBENTON; Vita beati Joannis Francisci Regis [...] in latinum sermonem conversa, Pragae 1718. (Erstausgabe: Paris 1716); vgl. SOMMERVOGEL (wie Anm. 21), II, Sp. 1832 ${ }^{26}$ So z.B. Kazimierz WIERUsZEwsKI, Triumphus charitatis, Posnan 1717; Gabriel François LE JAY, Prima Joannis Francisci Regis, in: DERS., Bibliotheca Rhetorum. Tom. II, Paris 1725 S. 828-832; Descriptio sacri apparatus, Viennae 1738; Prima Joannis Francisci Regis, Claudiopoli 1738 .

${ }^{27}$ Descriptio sacri apparatus (wie Anm. 26), S. 4-5.
}

Jesuitische Emblemzentren und Autoren

am Anfang des 17. Jahrhunderts

Die ersten emblematischen Viten von Jesuitenheiligen entstanden als ..affixiones" seit der Wende vom 16. zum 17. Jahrhundert in den bedeutendsten Jesuitenkollegien Europas, am Anfang als anonyme Kollektivschöpfungen ${ }^{28}$ Die ersten Zentren bildeten sich in Italien ${ }^{29}$. Die Ordensgeschichten beschreiben zwar die Feste der einzelnen Ordenshäuser nicht näher, wir können jedoch voraussetzen, daß Rom und die italienischen Kollegien die größte Pracht entfaltet haben. Um diese Zeit war in Rom jener Fammiano Strada tätig, auf den sich mehrere jesuitische Emblematiker als auf ihren Meister beriefen. Stradas Symbole wurden zum Teil am gemeinsamen Versammlungsort der Marianischen Sodalitäten in Rom der Öffentlichkeit vorgestellt ${ }^{30}$.

Am Anfang des 17. Jahrhunderts zählten noch Mantua, Palermo, Neapel und Mailand zu den bedeutenden Zentren der Emblematik in Italien. In Mantua z.B wurden die Tugenden von Ignatius' „epigrammata et emblemata“ vorgeführt $^{31}$. In Palermo berichten die Quellen über Fammiano Stradas Wirken. Ein Jesuit aus Palermo und vermutlicher Schüler Stradas, Francesco Rajati, fertigte über Ignatius und Franz Xaver anläßlich ihrer Heiligsprechung (1622) eine Serie in ,symbola et inscriptiones“ an. In Neapel und Mailand wurden um 1622 Embleme über Franz Xaver angefertigt ${ }^{32}$.

Gleichzeitig mit der Tätigkeit der italienischen Jesuiten entstanden auch in Spanien Embleme über die Heiligen des Ordens. Deren Schöpfer waren nicht unbedingt Mitglieder der „Societas Jesu“, wie etwa der berühmte Sebastiàn

${ }^{28}$ PIETRASANTA, De symbolis (wie Anm. 18), S. 10-11; vgl. Julius Hortinus Roscius, Emblemata sacra S. Stephani Caelii Montis inter columniis affixa, Roma 1589

${ }^{29}$ Leonard von MATT - Hugo RAHNER, Ignatius von Loyola, Würzburg 1955, S. 123-126: Joseph Jouvancy, Historiae Societatis Jesu Pars Quinta [...] ab anno Christi MDXLI. ad MDCXVI, Romae 1710, S. 337-339; vgl. Menestrier, Philosophia imaginum (wie Anm. 15). S. 66 .

${ }^{30}$ Pietrasanta. De symbolis (wie Anm. 18), S. 10-11, 356; Sforza Pallavicino, Arte dello stile, Bologna 1647. S. 79-80; Jacobus Boschius, Symbolographia, Augustae Vindelicorum Dilingae 1701. S. 33.

"Annuae Litterae Societatis lesu Anni 1609, Dilingae s.a. S. 8-9.

${ }^{32}$ Fammiano STRADA, Saggio delle feste che si apparechiano nel Collegio Romano in Honore de Santi Ignatio et Francesco da N. S. Gregorio XV. canonizati, Roma 1622 (weitere Ausgabe Venetia 1622): Franciscus R AJAT. Symbola et Inscriptiones adornatae honori SS.

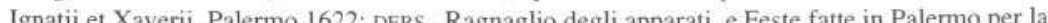

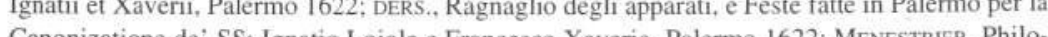
Canonizatione de' SS; Ignatio Lojola e Francesco Xaverio, Palermo 1622; MENESTRIER, Philosophia imaginum (wie Anm. 15), S. 234, 357, 608 . 
de Covarrubias Orozco aus Toledo oder jener Alonso Ledesma y Buitrago aus Segovia, der über Franciscus Borgia und Ignatius von Loyola Embleme schuf $^{33}$.

Als man sich im römischen Zentrum des Ordens nach der Seligsprechung Ignatius' auf seine Heiligsprechung vorbereitete, beschloß man, die emblematische Form auch in andere europäische Länder zu verpflanzen. Zu diesem Zweck schickte man die besten Emblematiker aus den Reihen der römischen Jesuiten ins Ausland. Die Seligsprechung von Ignatius hat man in Brüssel wie in mehreren Kirchen der niederrheinischen Ordensprovinz (z.B in Köln und Trier) unter anderem durch das Aushängen von Emblemen gefeiert ${ }^{34}$. In dieses Gebiet schickte man zuerst Fammiano Strada, später seinen Schüler Silvestro Pietrasanta. Strada hat vor allem die Kollegien der flämischbelgischen Ordensprovinz (Leuven, Brüssel u.a.), Pietrasanta die der norddeutschen Ordensprovinz mit den Symbolen und ihrem Gebrauch bekannt gemacht $^{35}$.

Wenn die Heiligsprechung des Ordensgründers und die Franz Xavers im Jahre 1622 in den Jesuitenkollegien bereits europaweit auch emblematisch gefeiert wurde, dann ist dies zum großen Teil durch Stradas und Pietrasantas Wirken zu erklären. Über den dritten General des Ordens, den 1624 seliggesprochenen Franciscus Borgia wurden ebenfalls Embleme gefertigt. Die Form, in der man die Embleme in Köln aushängte, wurde zur Regel und bei den jesuitischen Heiligsprechungen bis zur Mitte des 18. Jahrhunderts europaweit angewandt ${ }^{36}$.

Unter den niederländischen Emblemzentren waren Antwerpen, Brüssel und Tournay die bedeutendsten ${ }^{37}$. Von den jesuitischen Emblemzentren Frankreichs sollen hier Avignon, La Flèche und Pont-à-Mousson ${ }^{38}$, von

${ }^{33}$ Alonso Ledesma y ButrRago, Epigramas y Hieroglíficos [...] excelencias de Santos. Madrid 1625.

${ }^{34}$ Annuae [...] 1609, S. 26-28; Fridericus REIFFENBERG, Historia Societatis Iesu ad Rhenum Inferiorem e MSS. Codicibus, Tom. I, Coloniae 1764, S. 466.

${ }^{35}$ Imago Primi Saeculi (wie Anm. 24), S. 731; MeNESTRIER. Philosophia imaginum (wie Anm. 15), S. 33 .

${ }_{37}^{36}$ ReIFFEnBerg, Historia (wie Anm. 34). S. 531-534.

${ }^{37}$ Typus mundi, Antverpiae 1627; Guillelmus Hestus, Emblemata sacra de Fide, Spe, Charitate, Antverpiae 1636; VINCARTIUS, Sacrarum (wie Anm. 24); MENESTRIER, Philosophia imaginum (wie Anm. 15), S. 93; Jacob MASEN, Speculum imaginum veritatis occultae, Coloniae 1681, S. $543-544$.

${ }^{38}$ Menestrier, Philosophia imaginum (wie Anm. 15), S. 225, 541, 743-744; Jennifer MoNTAGU, The Painted Enigma and French Seventeenth Century Art, in: Journal of the Warburg and Courtauld Institutes 31 (1968), S. 307-335, hier: S. 307. denen Polens Kalisch (Kalisz) und Posen (Poznan) ${ }^{39}$, von denen in Ungarn Pozsony (Preßburg) und Nagyszombat (Tyrnau) hervorgehoben werden ${ }^{40}$. In Kalisch erschien einer der ersten emblematischen Drucke, die den Kult von heiligen Jesuiten verbreiten sollten ${ }^{41}$.

Klassische Beispiele der emblematischen Darstellung von heiligen bzw. zur Kanonisation vorgesehenen Gestalten unter den Jesuiten sind in der Imago primi saeculi aus der Brüsseler Werkstatt enthalten. Die Embleme über die Heiligen und Märtyrer des Ordens, die dem fünften, „Societas honorata..." betitelten und die hervorragenden Jesuiten aufzählenden Buch beigefügt wurden, sollten dazu dienen, durch die in der Darstellung versteckte similitudo die Herrlichkeit (gloria) der einzelnen Personen, den Ruhm ihrer Heiligkeit (fama sanctitatis), ihre Würde (dignitas), Ehre (honor), Ansehen (existimatio) und Seligkeit (felicitas) zu preisen (ornavere) (2) $^{42}$ Im wesentlichen war es die in der Imago primi saeculi angewandte Methode, die die Jesuitenemblematik maßgeblich beeinflußt und die Gattungsgeschichte der emblematischen Heiligenviten bis zur Mitte des 18. Jahrhunderts weitgehend geprägt hat.

\section{Quellentypologie, Autorschaft und Publikationsgeschichte}

Jene Drucke, die Embleme und Emblemserien über die Heiligen bzw. die nach ihrem Tode besonders verehrten Mitglieder des Ordens enthalten ${ }^{43}$, stehen etwa zu drei Viertel mit einem konkreten Anlaß im Zusammenhang, und nur der Rest ist als von solchen Ordensereignissen unabhängige, selbständige Veröffentlichung entstanden. Die durch Ordensereignisse veranlaßten Werke lassen sich in drei Gruppen unterteilen:

${ }^{39}$ Vgl. Paulina BuCHWALD-PeLCowA, Emblematy w drukach polskich i polski dotyczacych XVI-XVIII wieku. Bibliografia, Wrocław - Warszawa - Krakow - Gdansk - Lodz 1981.

${ }^{t 0}$ Emericus TolvaY, Ortus et progressus almae, Archi-Episcopalis Societatis Iesu Universitatis Tyrnaviensis [...] ad annum usque MDCLX, Tyrnaviae 1725, S. 76, 90-91.

${ }^{41}$ Encomia sive laudationes beatorum Stanislai Costca et Aloisii Gonzaga, Calisii 1606.

${ }^{42}$ Imago Primi Saeculi (wie Anm. 24).

${ }^{43}$ Die bibliographische Arbeit wurde noch nicht abgeschlossen. Im Vergleich zur Materialsammlung von G. Richard Dimler wurde die Zahl der Titel bisher um etwa $30 \%$ erhöht. Vgl. G. Richard Dimler. Short Title Listing of Jesuit Emblem Books, in: Emblematica 2 (1987), S. 139-187. 
1. die Veröffentlichungen aus Anlaß von Selig- bzw. Heiligsprechungen (etwa $35 \%$ ), in denen die im jeweiligen Ordenshaus nach der Kanonisation veranstalteten Feierlichkeiten in ihren denkwürdigsten Momenten dokumentiert werden ${ }^{44}$;

2. Werke, die die Heiligen unabhängig von ihrer Kanonisation verherrlichen (etwa $15 \%$ ), wobei die darin enthaltenen emblematischen Viten verschiedene lokale Ereignisse, z.B Ordensjubiläen, die Einweihung der zur Verehrung des Heiligen gebauten Kirche oder die Ankunft seiner Reliquie repräsentieren ${ }^{45}$;

3. die sog. libri gradualia, die an den Jesuitenuniversitäten und Akademien anläßlich der Prüfungen verteilt wurden $(25 \%)^{46}$.

Unter den Veröffentlichungen, die unabhängig von konkreten Anlässen erschienen, lassen sich folgende Haupttypen unterscheiden:

1. Veröffentlichungen, die ausschließlich die emblematische Heiligenvita enthalten ${ }^{47}$;

2. Veröffentlichungen, die mehrere Schriften desselben Autors, darunter eine emblematische Heiligenvita, bringen ${ }^{48}$;

3. Veröffentlichungen, die thematisch verwandte Werke von zwei oder mehr Autoren aufführen - in diesen treten die Emblemserien zusammen mit Texten anderer Gattungen (wie z.B. Predigten, gereimte Elogien-Zyklen, Heiligenviten in Prosa und Schuldramen) auf ${ }^{49}$;

4. Veröffentlichungen, die nur nebenbei, sozusagen als Füllsel Embleme bringen $^{50}$;

${ }^{4}$ Z.B. Symbola et Emblemata in Canonizatione SS. Aloysii et Stanislai, Cremsii 1727: Johannes Baptista MAYR, Sacra solemnia, quibus divos suos, Aloysium Gonzagam, et Stanislaum Kostkam, Jaurini 1728 .

${ }^{45}$ Z.B. Albert INES, S. Franciscus Xaverius Indiarum apostolus [...] vitae sancti gesta repraesentantibus illustratus, Posnaniae 1649.

to Z.B. Gabriel HevenesI, Academicus Viennensis sive B. Stanislaus Kostka, Viennae 1690; DERS., S. Ephebus sive B. Aloysius Gonzaga, Viennae 1690; Querck, Acta (wie Anm. 24), Ein Exemplar des Werkes von Querck ohne Embleme befindet sich in der UB Budapest, Sign. Ac 5404.

${ }^{47}$ Z.B. Carlo Bovıo, Ignatius insignium. epigrammatum et elogiorum centuriis expressus, Romae 1655 .

${ }^{48}$ Z.B. Antonius MAURISPERG, Floralia sacra, seu conceptus symbolici e floribus collecti, in: DERS., Opuscula varia, Steyr 1726, S. 1-42.

${ }^{49}$ Z.B. Symbola in tractu Collegij, in: Dij Gemelli [...] sive Divi Aloysius Gonzaga, ac Stanislaus Kostka, Tyrnaviae 1727.

${ }^{50}$ Z.B. Imagines emblematicas, in: Carolus Libertinus, Divus Franciscus Xaverius, Pragae 1673, f. 13a-18a.
5. Emblemkompendien und -lehrbücher. Die letzte Gruppe haben wir nur deswegen in die Untersuchung mit einbezogen, weil man mit ihrer Hilfe eine Reihe von emblematischen Heiligenviten des Jesuitenordens rekonstruieren kann, die selbständig nie im Druck erschienen sind ${ }^{51}$.

Ein Teil der Werke (etwa 20\%) sind anonyme Veröffentlichungen. Diese stehen ausnahmslos mit einem Jesuitenkolleg (z.B Antwerpen, Bourges, Brüssel, Dillingen, Graz, Kalisch, Kolozsvár [Klausenburg], Krems, München, Trencsén [Trentschin], Vilnius, Warschau, Wien und Wrocław [Breslau]) in Zusammenhang. Um den Kreis der potentiellen Autoren näher zu bestimmen, müssen wir in jedem Fall alle Ordensmitglieder in Betracht ziehen, die im jeweiligen Kolleg zur Erscheinungszeit der ersten Ausgabe lebten und tätig waren. Um so mehr, als die Versuche der früheren Bibliographien, die Autoren der anonymen Werke zu identifizieren, manchmal zu falschen Angaben führ$\operatorname{ten}^{52}$

Jene Veröffentlichungen, deren Autoren bekannt sind und die etwa vier Fünftel des gesamten Corpus ausmachen, sind zu $70 \%$ von Jesuiten verfaßt worden. Nur wenige unter diesen verfaßten mehr als ein Werk, und der größte Teil der Werke ist nur ein einziges Mal verlegt worden - ein Hinweis auf den Gelegenheitscharakter der Veröffentlichungen und die bestimmende Rolle des Ordens als Auftraggeber. Die Nichtjesuiten unter den Autoren sind etwa mit $10 \%$ im gesamten Material vertreten.

Vom Anfang des 17. Jahrhunderts bis zur zweiten Hälfte des 18. Jahrhunderts sind über die folgenden Jesuiten Embleme und Emblemserien verlegt worden: Ignatius von Loyola, Franz Xaver, Franciscus Borgia, Stanislaus Kostka, Aloysius Gonzaga, Franciscus Regis, die japanischen Märtyrer, Joannes Berchmann, Petrus Canisius, Roberto Bellarmino. Den ersten Seligsprechungen (Aloysius Gonzaga: 1605, Ignatius Loyola: 1609, Franz Xaver:

\footnotetext{
Menestrier, Philosophla imaginum (wie Anm. 15).

${ }^{2}$ So wurde z.B. das anonyme Werk Lilietum Aloysianum Rosetum Stanislaianum, Tyrna viae 1727, durch Sommervogel (wie Anm. 21), I, Appendix VIII. und nach ihm durch Dimlek. Short Title Listing (wie Anm. 43), S. 142. György Arvai zugesprochen. Den emblematischen Teil des Druckes folgt eine Predigt von László Turóczi, die in der Jesuitenkirche von Nagyszombat am 3.9.1727 gehalten wurde. Im Manuskript Annuae Collegij Tyrnaviensis S. J. (UB

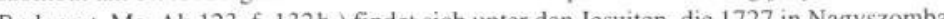

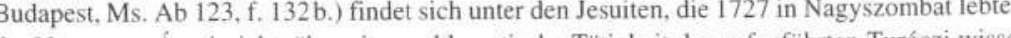
der Name von Arvai nicht, über eine emblematische Tatigkeit des aufgefuhrten Turóczi wissé wir nichts. Zugleich findet sich in dieser Liste der Name von János Gyalogi, der auch in der Beschreibung der Feierlichkeiten namentlich erwähnt wird (Annuae, f. 137a-138a.). Die Emblembücher von Gyalogi sind auch in der internationalen Fachliteratur registriert, und die Embleme der erwähnten anonymen Publikation wurden höchstwahrscheinlich von ihm gemacht.
} 
1619) waren nur wenige emblematische Veröffentlichungen gewidmet. Ein Kalischer Druck, der 1606 zur Seligsprechung Gonzagas erschienen ist, verherrlicht Gonzaga zusammen mit Stanislaus Kostka ${ }^{53}$. Die Emblemserien, die zur gemeinsamen Heiligsprechung von Ignatius von Loyola und Franz Xaver entstanden sind, dokumentieren durch ihre Entstehungsorte (Rom, Palermo, Venedig, Augsburg, Segovia) den Verbreitungsweg der Gattung, ihre Ausstrahlung vom engeren italienisch-spanischen Zentrum auf das deutsche Sprachgebiet.

Die größte Zahl der zwischen 1622 und der Heiligsprechung des Franciscus Borgia (1671) veröffentlichten Emblemserien ist Ignatius und Franz Xaver gewidmet. Die in dem durch Bourges, Prag, Posen und Wien begrenzten Gebiet erschienenen Drucke zeugen gleichermaßen für die Verbreitung der Gattung wie für die Verbreitung des jesuitischen Heiligenkultes. Nimmt man zu dieser Gruppe jene Veröffentlichungen hinzu, die im Zusammenhang mit Franciscus Borgias Heiligsprechung im Gebiet zwischen Avignon, Bourges, Rouen, München, Rom, Wien und Graz gedruckt wurden, dann hat man die größte Verbreitung der emblematischen Heiligenviten in der zweiten Hälfte des 17. Jahrhunderts vor sich, als die Gattung zugleich ihre höchste Blütezeit erlebte.

Die emblematische Ausstrahlung der Heiligsprechungen im 18. Jahrhundert (Aloysius Gonzaga und Stanislaus Kostka: 1726, Franciscus Regis: 1737) war vom Ausscheiden der west- und südeuropäischen Gebiete aus diesem Prozeß geprägt. Die Veröffentlichungen, deren Zahl jetzt zunahm und die ca. $35 \%$ des gesamten Corpus ausmachten, erschienen innerhalb eines relativ engen geographischen Raumes, auf deutschem, tschechischem, polnischem und ungarischem Sprachgebiet. Wenn vor der Kanonisation von Stanislaus Kostka in Neapel (1720) und in der Zeit um die Heiligsprechung des Franciscus Regis in Lille und Paris emblematische Viten erschienen - wobei letztere wohl auf die französische Abstammung des Heiligen zurückzuführen sind -, dann waren diese nur noch Ausnahmen.

Kupferstiche wurden nur den anspruchsvolleren Veröffentlichungen beigefügt. Die meisten Stiche wurden von unbekannten Stechern im Dienste der Jesuitendruckereien angefertigt, manchmal jedoch tauchen bekannte Graphiker und Kupferstecher (wie z.B Guillaume Chasteau, Lodovico Gimignani, J. C. Schalckh, Cornelius Galle, Wolfgang Kilian, Giovanni Martino Lerch u.a.) unter den Meistern auf. Aufgrund der Explikationen können wir in meh-

\footnotetext{
${ }^{53}$ Encomia (wie Anm. 41). 1606
}

reren Fällen eine aktive Beteiligung der Autoren an der Entstehung der Illustrationen voraussetzen. Die unbebilderten Veröffentlichungen machen etwa $65 \%$ des gesamten Materials aus, was zum Teil durch die bescheidenen finanziellen Möglichkeiten der einzelnen Kollegien und Mäzene, die die Veröffentlichung veranlaßten, bedingt ist, zum Teil aber dadurch zu erklären ist, daß die Jesuiten die Beifügung des Emblembildes nicht als unentbehrlich betrachteten.

\section{Strukturelle Eigentümlichkeiten und Funktionen}

Als Serie galt für uns jede Komposition, die sich auf den Lebensweg von Heiligen bezieht, nach chronologischen oder anderen Prinzipien geordnet ist und aus mehr als zwei selbständigen emblematischen Strukturen besteht. Gelegentlich zogen wir für die Untersuchung auch einzelne, serienmäßig nicht geordnete Embleme heran, die je eine Szene aus dem Leben der Heiligen, eine ihrer Tugenden oder Wundertaten bzw. einzelne Momente ihres Kultes illustrieren.

Im Aufbau der Serien lassen sich zwei verschiedene Konzeptionen unterscheiden. Bei dem einen Typus bilden die Ereignisse des Lebensweges das Grundprinzip der Anordnung, bei dem anderen hingegen die Tugenden. Beide Konzeptionen wurden seit Anfang des 17. Jahrhunderts kontinuierlich angewandt.

Die Struktur der einzelnen Embleme zeigt wenig Abwechslung. Die Form, die sich aus der knappen Beschreibung des biographischen Momentes, dem Motto (Lemma), dem Bild oder der Bildbeschreibung und der Explikation in Versen oder in Prosa zusammensetzt, ist seit dem Beginn des 17. Jahrhunderts allgemein üblich. Einigermaßen davon abweichend sind die Heiligenembleme der Emblemkompendien und -lehrbücher gestaltet. Neben Bild (Bildbeschreibung) und Motto werden hier in der belehrenden Explikation in Prosa, entsprechend der Funktion dieser Werke, Ursprung und Sinn des bildlichen Ausdrucks und des Lemmas erklärt, bzw. eine Begründung geliefert, warum die Darstellung zur Charakterisierung des Heiligen geeignet ist (similitudo, transfictio). Dieses Schema wollte Carlo Bovio dem Unterricht besser anpassen, indem er Lemma und Bild durch Multiplizierung der Explikation in drei verschiedenen literarischen Formen (Prosa, Epigramm und Elogium) erörterte ${ }^{54}$.

\footnotetext{
${ }^{54}$ Bovio, Ignatius insignium (wie Anm. 47)
} 
Von den beiden Grundtypen der Serien war der auf dem Lebensweg beruhende beliebter. Innerhalb dieses Typus können wir drei Varianten unterscheiden:

1. die Serie folgt der Vita des Heiligen und zeigt die Stationen seines Lebens von der Geburt bis zum Tod - solche Serien wurden sowohl für Selig-, als auch für Heiligsprechungen angefertigt ${ }^{55}$;

2. öfter als die erste Lösung wurde die ,vita et miracula“-Konzeption zur Anordnung der Embleme benutzt ${ }^{56}$;

3. die ,vita et miracula“-Anordnung wurde gelegentlich durch die Ereignisse der Kanonisation erweitert ${ }^{57}$.

In dem anderen, von den Tugenden der Heiligen ausgehenden Grundtypus der Serien lassen sich ebenfalls mehrere Varianten unterscheiden:

1. die Serie bringt zuerst die Bekehrungsszene und führt dann die Tugenden des Heiligen vor ${ }^{58}$;

2. in mehreren Veröffentlichungen wurden ausschließlich die Tugenden des jeweiligen Heiligen zu einer Serie zusammengestellt ${ }^{59}$;

3. den Tugenden wurden in einigen Fällen sog. miracula-Embleme beigefügt $^{60}$.

Neben den beiden, auf der Vita bzw. auf den virtutes beruhenden Grundtypen bildete sich noch eine dritte, gemischte Anordnungsmethode heraus. Bei dieser besteht die Serie aus Vita-, virtutes- und miracula-Emblemen, die gelegentlich durch die Darstellung der mit der Kanonisation zusammenhängenden Ereignisse ergänzt werden ${ }^{61}$.

Die oben angeführten Anordnungstypen und ihre Varianten sind unabhängig von der Zahl der Embleme, sie stehen jedoch in engem Zusammenhang mit den Funktionen der Serien. Die nach den Viten zusammengestellten Serien hatten vor allem das Ziel, den Heiligen bekannt zu machen und seinen Kult zu verbreiten. Die virtutes-Kompositionen hingegen hatten eine kom-

${ }^{55}$ Hevenesi, Academicus (wie Anm. 46); Descriptio, 1738 (wie Anm. 26).

${ }^{56}$ LEDESMA Y BUITRAGo, Epigramas (wie Anm. 33); Lilietum (wie Anm. 52), f. A2a.

${ }_{7}^{7}$ MAURISPERG, Floralia (wie Anm. 48), S. 1-42.

${ }^{88}$ Imago Primi Saeculi (wie Anm. 24), S. 714-719; Carlo Bovı, Rhetoricae suburbanum. Romae 1676, S. 2-9.

${ }^{59}$ Leopold GRUEBER, Honores sacri divis Aloysio Gonzaga et Stanislao Kostkae, Viennae

1727; Joannes DESPOTOVICH, Apparatus emblematicus, Viennae 1671.

${ }^{60}$ Imagines emblematicas (wie Anm. 50), f. I3 a-I8 a.

${ }^{61}$ Bovio, Ignatius insignium (wie Anm. 47): QuerCK. Acta (wie Anm. 24). plexere Funktion, nämlich die Vermittlung des aktiven, handelnden Kultes ${ }^{62}$. Jene Veröffentlichungen schließlich, die dem gemischten Vita-virtutes-Typus zuzuordnen sind, betonen neben der Darlegung und Einprägung der nachahmungswürdigen Beispiele die belehrende, pädagogische Funktion ${ }^{63}$.

Einige Veröffentlichungen verfolgten darüber hinaus noch ein weiteres Ziel. Die öffentlich ausgestellten, für konkrete Anlässe angefertigten Serien wurden zusammen mit ihrer Rahmenkomposition verlegt. Diese Beschreibungen dienten durch die ausführliche Darstellung der repräsentativen Elemente einer allgemeineren, über den jeweiligen Heiligen hinausgehenden Verbreitung des Kultes ${ }^{64}$.

\section{Verhältnis zwischen Bild und Text; die Quellen}

Die Autoren, die Embleme zu den Viten der Jesuitenheiligen verfaßten, haben sich einer besonderen Methode bedient. Diese Methode bestand im wesentlichen darin, daß man die Motive der humanistischen Emblematik des 16. Jahrhunderts übernahm und neu interpretierte, wobei man sie den Kanonisationsvorstellungen des Jesuitenordens und seinen weiteren Zielsetzungen gemäß adaptierte, bearbeitete, integrierte und kombinierte. Die Verbindung zwischen den Emblemen und den Ordenszielen wurde hergestellt, indem man die von den Humanisten edierte antike Literatur mit einbezog und sie bewußt adaptierte. Jene klassische literarische Bildung, die von der Ratio Studiorum vorgeschrieben wurde ${ }^{65}$, hat man sowohl im Bildmaterial der Embleme als auch in den Motti und den Explikationen verarbeitet. Auch konnten dadurch die Embleme ohne größere Schwierigkeiten verstanden und rezipiert werden.

Als Folge dieser Bestrebungen können wir von einem zweifachen Vermittlungsprozeß sprechen. Zum einen hat die jesuitische Heiligenemblematik die bei den Humanisten beliebten, klassisch-weltlichen Motive christianisiert, zum anderen vermittelte sie die Ideen des Ordens einem breiteren Publikum

\footnotetext{
${ }^{62}$ GRUEBer, Honores (wie Anm. 59), S. 22.

Bovio, Ignatius insignium (wie Anm. 47), f. 5 b.

${ }^{64}$ Lilietum (wie Anm. 52), f. A 2b. Vor dem Hauptaltar wurde eine Nachbildung der Höhle von Manresa des Ignatius aufgestellt, wo die Exerzitien entstanden sind. Symbola in tractu, f. D 1a-D 2b.

${ }^{65}$ Ratio atque Institutio Studiosorum Societatis Iesu (1586, 1591, 1599), Ed. Ladislaus Lukács, Romae 1986.
} 
Wir haben die Motti jener aus hundert Emblemen bestehenden Serie, die von dem bisher nicht zu den Emblematikern gezählten Antonius Maurisperg für die Heiligsprechung des Stanislaus Kostka angefertigt wurde (1726), mit dem Mottoregister im Handbuch von Henkel-Schöne verglichen. Aufgrund des Verhältnisses zwischen Motto, Bild und Bedeutung lassen sich die Embleme in vier Gruppen unterteilen:

1. zum selben Motto gehört dasselbe oder ein ähnliches Bildmotiv, die Bedeutung ist jedoch unterschiedlich ${ }^{66}$;

2. mit demselben Motto sind jeweils andere Bilder und Aussagen verknüpft. Das auf eine bildliche Ähnlichkeit zurückführbare Grundmotiv der unterschiedlichen Bedeutungen ist jedoch identisch ${ }^{67}$;

3. dasselbe, mit jeweils anderen Bildmotiven kombinierte Motto trägt eine ähnliche Bedeutung ${ }^{68}$;

4. mit demselben Motto sind jeweils verschiedene Bilder und Bedeutungen verknüpft ${ }^{69}$.

Unter den Emblembildern von Maurisperg kommen mehrere antike, literarische und mythologische Darstellungen vor. Da diese von den Jesuiten nur relativ selten benutzt wurden, ist anzunehmen, daß Maurisperg seine Kenntnise über humanistische und späthumanistische Emblematik zum Teil aus selbständigen Werken geschöpft hat. Als Beispiel dafür soll hier das Motiv „Herakles tötet zwei Schlangen in der Wiege“ näher betrachtet werden.

Bei Maurisperg liegt Herakles mit einem Lammfell zugedeckt in der Wiege. Die eine zerrissene Schlange erblickt man in seinen Händen, die andere in Stücken neben der Wiege ${ }^{70}$. Die Darstellung weicht von der mythologischen Erzählung über Heras Rache insofern ab, als Herakles hier nicht neben seinem Bruder auf einem eisernen Schild liegt. In der Explikation wird Stanislaus Kostka mit dem Kind Herakles verglichen, da er bereits im Kindesalter durch seine Tugenden hervortrat. Früher wurde das Motiv von Zincgref zum Aus-

${ }^{66}$ MAURISPERG, Vita (wie Anm. 22), S. 11; Arthur HENKEL - Albrecht SCHÖNE, Emblemata Handbuch zur Sinnbildkunst des XVI. und XVII. Jahrhunderts, Sonderausgabe, Stuttgart, 1978, Sp. 146.

${ }^{67}$ Maurisperg, Vita (wie Anm. 22). S. 19; Henkel - SCHÖNe (wie Anm. 66), Sp. 714; Andreas ALCIATUS, Emblemata, Lugduni 1566, S. 34, 186.

${ }^{67}$ Maurisperg, Vita (wie Anm. 22), S. 17; Henkel - Schöne (wie Anm. 66), Sp. 1296.

${ }^{69}$ Maurisperg, Vita (wie Anm. 22), S. 96; HENKEL - SCHÖNE (wie Anm. 66), Sp. 349; Bovı, Ignatius insignium (wie Anm. 47), Nr. 85.253: MENESTRIER, Philosophia imaginum (wie Anm. 15), S. 571-573.

MAURISPERG, Vita (wie Anm. 22), S. 6. druck der angeborenen Tugend, von Saavedra Fajardo zur Vorführung der angeborenen Tapferkeit der Fürsten benutzt ${ }^{71}$. In jesuitischen Emblembüchern und -kompendien kommt die Szene nicht vor, so daß das Motiv bei Maurisperg vermutlich nicht aus der jesuitischen Emblematik geschöpft wurde.

Auffallend viele Szenen und Motive aus der antiken Mythologie verwendet Ignatius Querck in seiner emblematischen Vita Loyolas. Zu den hundert Emblemen, die mit je einem der illustrierten biographischen Ereignisse in Parallele gestellt sind, wurden die Motti ausschließlich aus Werken verschiedener Klassiker (Ovid, Vergil, Claudian, Lukian, Juvenal, Horaz, Lukrez, Properz, Tibull, Seneca) gewählt, und fünfundzwanzig Emblembilder gehen auf mythologische Szenen zurück. So stellt z.B. der aus Troja fliehende Aeneas, der seinen Vater auf dem Rücken trägt und seinen Sohn Ascanius mit der Flamme über dem Kopf an der Hand führt, bei Querck jenes Ereignis aus dem Leben des Ignatius dar, als er ein Meßopfer darbrachte und die Anwesenden ein Feuer über dem Haupt des Heiligen erblickten. Das Motiv des mit seinem Vater fliehenden Aeneas findet sich sowohl bei Alciatus als auch bei Laurentius Haechtanus und Horozco y Covarrubias. Alciatus führt mittels dieses Emblems die kindliche Liebe vor ${ }^{72}$.

Die zweite grundlegende Quelle der Darstellungen bilden neben dem von den Humanisten vermittelten Bildungsgut die Emblemkompendien der Jesuiten. Jakob Masen z.B. sagt in seinem Speculum imaginum veritatis occultae, der Adler sei vortrefflich in der emblematischen Darstellung der Heiligen zu verwenden. Johannes Baptista Mayr hat Masens Schrift wahrscheinlich gekannt, denn in seinem Werk zur Lobpreisung der neuen Jesuitenheiligen Aloysius Gonzaga und Stanislaus Kostka hat er Masens Empfehlung des Adlermotivs zur führenden Idee erhoben ${ }^{73}$.

Arbeitsmethode im Spiegel der Anwendungstypen der Bildmotive

1. Demselben Motiv werden in der Darstellung des Heiligen jeweils andere Bedeutungen zugeordnet. In den emblematischen Viten des Ignatius von Loyola begegnet man oft Motiven wie dem des bolis bellica, der in der Luft explodierenden feurigen Zündkugel, die auf das Soldatenleben des Heiligen

\footnotetext{
${ }^{71}$ Henkel - Schóne (wie Anm. 66), Sp. 1641-1642.
${ }^{72}$ Querck, Acta (wie Anm. 24), S. 83; HenKel- SchONe (wie Anm. 66), Sp. 1703.

${ }^{73}$ MASEN, Speculum (wie Anm. 37), S. 859; MAYR, Sacra solemnia (wie Anm. 44).
} 
hinweisen. In der Imago primi saeculi drückt das Motiv zusammen mit dem Motto „Sursum rapit ignis“ die Elevation des Ignatius während des Betens aus. Bei Bovio steht es zusammen mit dem Motto „Ardendo concussit“ für den Akt, mit dem der Heilige sich Gott empfahl. Menestrier verwendet das Motiv mit dem Motto „Percussus concipit ignes“ für seine Verwundung und Bekehrung, Querck mit dem Motto „Inde tremit tellus. Ovid. Metam. 5." für das himmlische Licht, das sich infolge des nächtlichen Gebets des Ignatius auf das Elternhaus senkte. In allen vier biographischen Momenten läßt sich eine gewisse Ähnlichkeit mit dem bolis bellica nachweisen ${ }^{74}$.

Die Erklärung für jenen Prozeß, in dessen Verlauf das bildliche Motiv übernommen, durch neue Motti ergänzt und reaktualisiert wurde, findet sich in der Quelle des betreffenden Motivs. Diese Quelle haben die Jesuitenautoren, wenngleich von ihnen nicht ausdrücklich erwähnt, vermutlich selbst gekannt. Nach unseren Kenntnissen taucht das Motiv des bolis bellica im 3. Band der von Jacobus Typotius verfaßten Symbola divina et humana (1603) zum ersten Mal in emblematischem Kontext auf. Dort steht es für den ferraresischen Fürsten Alfons I., der bei Ravenna eine Metallkugel mit Schießpulver füllte und sie als Geschenk getarnt in das feindliche Lager schickte. Die Kugel explodierte, vernichtete in ihrer Umgebung alles und verhalf dem Fürsten zum Sieg. Das Emblem von Typotius entstand als Erinnerung an diesen Kriegserfolg. Das Motto („Loco et tempore“, „A lieu et temps“) stammt von Ariost und weist auf die Umstände hin, unter denen die Kugel abgeschickt wurde. Laut Kommentar sei das Motiv zur Darstellung von klugen, weisen und tugendhaften Menschen geeignet, und der Sinn des Mottos sei als die erneuerte Verwendung zweier Ovidscher Verse zu verstehen ${ }^{75}$.

Pietrasanta beruft sich, indem er das Emblem ebenfalls mit dem ferraresischen Fürsten Alfons I. verknüpft, weder auf Typotius noch auf den ihn kommentierenden Boodt, hält es jedoch auch allgemein zur Darstellung tugendhafter und weiser Personen geeignet. Nachdem das Motiv von Pietrasanta vermittelt, empfohlen und durch seine Interpretation den Ordenszwecken angepaßt wurde, brachten es die Jesuiten mittels neuer Motti mit Ereignissen aus dem Leben des Ordensgründers in Zusammenhang. Vor Pietrasanta war es unter anderem Covarrubias Orozco, der das Motiv zur Vorführung des

${ }^{74}$ Imago Primi Saeculi (wie Anm. 24), S. 719; Bovıo, Ignatius insignium (wie Anm. 47). Nr. 10. 28: MeNestrier, Philosophia imaginum (wie Anm. 15), S. 473; Querck. Acta (wie Anm. 24), S. 9 .

${ }^{5}$ Jacobus Typotius, Symbola divina et humana. Tom. I-III, Pragae 1601-1603, hier: Tom. III, S. 57, 60
Ruhmes und der Herrlichkeit benutzt hatte (1610). Nach Pietrasanta verwendeten die Jesuiten das Motiv auch zum Ausdruck anderer Inhalte. Laut Boschius (1701) sei das bolis bellica gleichermaßen geeignet, den seligen Tod der Heiligen, die Erreichung des Seelenheils und die menschliche Strebsamkeit (ambitio) darzustellen ${ }^{76}$

2. Ein ähnliches Motiv wird zur Darstellung von verschiedenen Ereignissen aus dem Leben der Heiligen benutzt. Dem Motiv des Wasser schöpfenden Engels begegnen wir in zwei, 1640 veröffentlichten, jesuitischen Emblemen. In der Imago primi saeculi steht es zusammen mit dem Motto ..Vis maxima, sed sine vinculo" für die Vollkommenheit der jesuitischen Lebensregeln. Zur gleichen Zeit führt Vincartius mit Hilfe des Motivs, das er in den Vordergrund einer mehrere narrative Momente vereinigenden Bildkomposition stellt, die innere Läuterung des Franz Xaver vor (das Motto dazu lautet: "Semper major erit, quantum se effunderit unda“). Das Motiv taucht außerdem in einer Serie zur Heiligsprechung von Franciscus Borgia auf, wo es die Mönchstugend der Selbstentleerung repräsentiert. Das Motto „Depressa implebitur" drückt aus, daß der Heilige, indem er sich entleert, von Tugenden erfüllt wird. Das Motiv des Wasser schöpfenden Engels versinnbildlicht sowohl in der Serie über Franz Xaver als auch in der über Franciscus Borgia einen auf Gegensatz beruhenden, spannungsvollen Gedanken: die Quelle, d.i. der Heilige, wird durch die Hingabe, das Ausströmen seines Selbst scheinbar weniger, in Wirklichkeit jedoch wird er bereichert und geläutert. Dieses Paradoxon bildet die Grundlage für die Aktualisierung der beiden erwähnten Motive, und so wird es von Bovio in einem Emblem über den Ordensgründer eingesetzt. Das Emblem stellt die Läuterung des Ignatius dar, der während seines Lebens am Hof die weltlichen Eitelkeiten verwirft. Das Motto - „Mergitur dum impletur" (Im Versinken füllt es sich) - sowie das Bild des im Brunnen untertauchenden Gefäßes stehen in engem gedanklichem Zusammenhang mit den erwähnten Emblemen über Xaver und Borgia ${ }^{77}$.

3. Für dasselbe biographische Motiv stehen verschiedene emblematische Lösungen. In den emblematischen Heiligenviten wiederholen sich meist die gleichen Motive, für die Embleme der Serien hingegen ist eine solche Wie-

${ }^{76}$ Pietrasanta, De symbolis (wie Anm. 18), S. 252; HeNKEL - SCHONE (wie Anm. 66), Sp. 1522: BoschIUs, Symbolographia (wie Anm. 30), Classis I. Nr. DCXXI. DCCCLXXXIX, Classis IV, Nr. V

77 Imago Primi Saeculi (wie Anm. 24), S. 174; VINCARTIUS, Sacrarum (wie Anm. 24), S. 132: Despotovich, Apparatus (wie Anm. 59). Nr. 57; Bovio, Ignatius insignium (wie Anm. 47), Nr. 3.7. 
derholung nicht immer kennzeichnend. Der individuelle Erfindungsreichtum der Verfasser konnte sich ja gerade im Finden unterschiedlicher Lösungen entfalten.

Ein wiederkehrendes Motiv der Serien über Ignatius ist die Szene ,Ignatius bekehrt einen Juden mit drei Worten“. Bovio vergegenwärtigte dieses Ereignis mit Hilfe des Mottos „Non mole, sed vi“ und der Gestalt der Remora, jenes mythologischen Wesens, das den Schiffen auf dem Meer den Weg verstellt. Querck verwendet das Bild des Magnetsteins, der das Herz anzieht, und das darauf hinweisende Motto „Poterit magnes non ducere ferrum? Proper 4.5.". Beide Verfasser betonen die übernatürliche Kraft, die den Worten des Ignatius innewohnt, und beide Embleme sind durch die Aktualisierung früherer Motive zustande gekommen ${ }^{78}$.

Die Remora (Hemmfisch) gehört zu den beliebtesten Motiven in der Emblematik des 16./17. Jahrhunderts. Mit ähnlicher Bildstruktur taucht es in unterschiedlichen Bedeutungen und mit verschiedenen Motti unter anderem bei Alciatus, Corrozet, Sambucus, Laurentius Haechtanus, Picinelli, Camerarius, Ferro de Rotarij, Aresi und Menestrier auf. Für Bovio haben vermutlich jene Emblematiker als Quelle gedient, die durch die Remora die Macht und die Kraft ausdrückten: Corrozet, Sambucus und Haechtanus ${ }^{79}$.

Das Motiv des Magneten, der das Herz anzieht, stammt ebenfalls aus dem 16. Jahrhundert: Georgia Montanea z.B benutzte es zur Versinnblildlichung der Gnade-Gottes-Idee. Sein Motto „Non tuis viribus“ weist auf dieselbe übernatürlich-göttliche Kraft hin wie das von Querck, nur daß er die Erscheinung vom „Herzen“, während Querck sie vom „Magnet“ ausgehend betrachtet ${ }^{80}$.

4. Dasselbe Ereignis tritt in den Emblemserien von verschiedenen Heiligen auf. Die Kanonisation wird in den Serien häufig dargestellt, und in den meisten Fällen wird sie durch Himmelskörper versinnbildlicht. Für mehrere Heilige wurde das Sternmotiv zum Ausdruck der Kanonisation gewählt. Querck stellt etwa die Heiligsprechung von Ignatius im Bild eines an den Himmel gesetzten neuen Sternes dar. 1727 wurde dann Quercks Emblem etwas modifiziert und auf Aloysius Gonzaga bezogen. Der unbekannte Verfasser übernahm das Motto, das Querck Ovids Metamorphosen entlehnte („Coelestibus

\footnotetext{
${ }^{7 \pi}$ Bovio, Ignatius insignium (wie Anm. 47), Nr. 80.238; QUERCK, Acta (wie Anm. 24). S. 78

HENKEL - SCHONE (wie Anm. 66), Sp. 712-713; BoschiUs, Symbolographia (wie Anm. 30), Classis I. Nr. DCCIV 1-2, Classis III. Nr. DV; Giovanni FERRO DE' RotARIJ, Theatro d'Imprese, Venetia 1623, S. 598-599.
}

${ }^{80}$ HeNKEL - SCHONE (wie Anm. 66), Sp. 82-83. intulit astris“), unverändert. Das Bild hingegen ist abgewandelt, indem der neue Stern nicht durch eine aus den Wolken ausgreifende Hand, sondern vom Genius des Papstes Benedikt XIII., der Gonzaga heiliggesprochen hat, an den Himmel gesetzt wird. Die Heiligsprechung von Franciscus Regis hat man ebenfalls in Form eines neuen Sternes am Himmel symbolisiert. Die sinnbildhafte Darstellung hervorragender Persönlichkeiten (Christus, Könige, Fürsten usw.) durch Sonne und Mond war bereits im 16. Jahrhundert allgemein verbreitet, und das Motiv des am Himmel erscheinenden neuen Sternes war ebensowenig unbekannt. Gabriel Rollenhagen z.B. benutzte es, um die Allmacht Gottes zu vergegenwärtigen ${ }^{81}$.

Außer den Himmelskörpern bediente man sich zur Darstellung der Kanonisation auch diverser irdischer Gegenstände. Bovio etwa versinnbildlicht die Heiligsprechung des Ordensgründers durch das brennende Feuer auf einem jesuitischen Wappenaltar und das Motto „sacratur et sacrat“. Um die Heiligsprechung von Stanislaus Kostka symbolisch darzustellen, benutzt Antonius Maurisperg in einer Serie das Bild des Blumenkorbs, der sich mit Lilien gefüllt von der Erde abhebt, in einer anderen das des starken Baumes inmitten einer stilisierten Landschaft. Ähnlich wie die Himmelskörper sollten diese Motive das Außerordentliche des Ereignisses hervorheben ${ }^{82}$.

5. Das gleiche Ereignis wird von verschiedenen Autoren mit Hilfe desselben Motivs vergegenwärtigt. Der Darstellung des Todes und der Ausmalung seiner Umstände widmeten die Jesuiten eine besonders große Aufmerksamkeit. In den Viten von Franz Xaver wird oft das Mondmotiv verwendet. Zur Versinnbildlichung seines Todes bezog man sich mehrmals auf das Motiv der Mondfinsternis (eclipsis Lunae), dem man zum Teil unterschiedliche Motti beifügte. In der Imago primi saeculi z.B. steht das Motto „Tum te terra teget, cum totum impleveris orbem" (die Erde bedeckt dich gerade, als du die ganze Welt erfüllst“), bei Picinelli hingegen heißt es: „Cursum haud sistit in umbra“ (im Schatten bleibt die Bewegung nicht stehen). Nach der Erklärung in den Explikationen ist das Bild der Mondfinsternis zur Darstellung des Todes von Franz Xaver geeignet, da es zum einen den Gegensatz zwischen der Dunkelheit des Todes und der schnellen und ruhmreichen Verbreitung des Rufes der Heiligkeit ausdrückt, zum anderen die Machtlosigkeit des Todes gegenüber

${ }^{81}$ Menestrier, Philosophia imaginum (wie Anm. 15), S. 225; QUerCK, Acta (wie Anm. 24). S. 100; Symbola in tractu (whe Anm. 49), f, a 2/b; Prima Joannis (wie Anm. 26), S. 36; HENKFISCHÖNE (wie Anm, 66), Sp. 41 .

${ }^{2}$ Bovio, Ignatius insignium (wie Anm. 47), Nr. 100.286: MAURISPERg. Floralia (wie Anm. 18), S. 19; MAURISPERG, Vita (wie Anm. 22), S. 100 
der Kraft der Heiligkeit vorführt. Das Motiv findet sich auch in Saavedra Fajardos Idea de un Principe (1640), wo es zusammen mit dem Motto „Censurae patet" Sinnbild des Fürsten ist. In Paolo Giovios Dialogo dell'imprese (1555) steht eine dem Emblem von Saavedra Fajardo ähnliche Beschreibung $^{83}$.

Im Kompendium von Boschius kann das Motiv der Mondfinsternis, mit verschiedenen Motti verknüpft, außer für den Tod gleichermaßen für Beständigkeit, Freundschaft, Glück, Tugend oder eine Fürstin stehen. Die Quellenangaben bei Boschius (Menestrier, Le Moyne, Bouhours u.a.) weisen zum einen auf die Beliebtheit des Motivs bei den Jesuiten hin, zum anderen bezeugen sie, daß die Provenienz des Emblems aus der humanistischen Tradition des 16. Jahrhunderts den Jesuiten bekannt war ${ }^{84}$. Zu Bouhours' Emblem (1671), das der Versinnbildlichung einer Fürstin dient, bemerkt nämlich Boschius, der ,ursprüngliche“ Schöpfer des Emblems sei „Ammirati" gewesen (Scipione Ammirato, 1562), Bouhours jedoch habe sein Emblem „,verbessert“ (,,a Bouhoursio sic emendatum“ “85. Aus alldem können wir schließen, daß das Motiv von dem bei Picinelli aufgeführten Kardinal Monte den Jesuiten vermittelt wurde, die es dann im Zusammenhang mit Franz Xavers Heiligsprechung verwendeten ${ }^{86}$

6. Die Übertragbarkeit der Motive auf andere Jesuitenheilige. Bei jenen Jesuitenheiligen, die in den Kanonisationsplänen des Ordens miteinander verknüpft, mit ähnlichen Eigenschaften ausgestattet und zur gleichen Zeit heiliggesprochen wurden, läßt sich das Verfahren beobachten, wonach die für den einen Heiligen bereits in Anspruch genommenen Motive auf einen anderen angewandt wurden.

Das Motiv der brennenden Kirche (sacra aedes flammis incensa) steht bei Pietrasanta, um zusammen mit dem Motto „Alterutra clarescere flamma“ den Ruhm von Aloysius Gonzagas Heiligkeit zu vergegenwärtigen. Laut Pietrasanta zierte diese Inschrift das einstige Heiligtum der Diana in Ephesos. Im 16. Jahrhundert verknüpfte man das Motiv mit einem anderen Motto (,,opes

${ }^{83}$ Menestrier, Philosophia imaginum (wie Anm. 15), S. 225; Imago Primi Saeculi (wie Anm. 24), S. 721; MENESTRIER, ebd. S. 311: Filippo PICINELLI, Mondo simbolico, Milano 1669 S. 39-40. (Erstausgabe: Milano 1653); Filippo PICINELLI - Augustinus ERATH, Mundus symbolicus, Coloniae 1687, S. 45-46; HeNKEL - SCHÖNe (wie Anm. 66), Sp. 35-36.

${ }^{4}$ Boschius, Symbolographia (wie Anm. 30), Cl. III. Nr. DCCLXXVIII. Nr. CCCXXXVIII,

Cl. II. Nr. 95, Cl. III. Nr. CDXVI. Nr. MCXXIV, Cl. II. Nr. 465, Nr. 449.

${ }^{85}$ Ebd., Cl. II. Nr. 449, Scipione AmmiRATo, Il rota overo dell' imprese, Napoli 1562.

${ }^{86}$ PicinelLi - ERATH, Mundus symbolicus (wie Anm. 83), S. 45-46. non animum"). Nach Ruscelli und Typotius war es ursprünglich auf die italienische Adlige Ersilia Cortese de Monti bezogen und sollte deren Beständigkeit, Stärke und unbesiegbare Seele vorzeigen. Das Motto war einem Vers in Senecas Medea entnommen (,Opes fortuna auferre, non animum potest"). Typotius brachte das Bild und das Motto mit einer anderen italienischen Fürstin, mit Felice Ursina Columna Ducissa Paliani e Tagliacozae, in Zusammenhang. Bovio wiederum ergänzte das Motiv durch das Motto „Ferte citi fontem“ und wandte es statt auf Aloysius Gonzaga auf Stanislaus Kostka an. Er führte damit die Szene vor, als Stanislaus in das eiskalte Wasser tauchte, um sein vom Feuer der göttlichen Liebe entflammtes Herz zu erquicken. Das Emblem wurde in dieser Form immer wieder auf Kostka angewandt, so etwa in Trentschin, als man dort die Heiligsprechung Gonzagas und Kostkas feierte ${ }^{87}$.

Wir können aber auch den umgekehrten Prozeß beobachten, daß ein ursprünglich mit Stanislaus Kostka verbundenes Motiv auf Aloysius Gonzaga übertragen wurde. Das Bild der unreifen Weintraube, die in einem Glasgefäß frühzeitig zur Reife gebracht wird (botrus phialae perspicuae inclusus), bezog sich bei Pietrasanta noch auf den Tempelgang Mariä, in der Imago primi saeculi dagegen, mit einem zum Teil abgeänderten Motto (,.Ut cito maturescat"), bereits auf Stanislaus Kostka und seinen Eintritt in den Orden. Bovio änderte das Motto (,Educatus non educitur") und wandte so das Motiv auf Aloysius Gonzaga an, um den kraft des häufigen Betens gereiften Heiligen darzustellen ${ }^{88}$. Wie aus alldem hervorgeht, gilt die für die Heiligenviten charakteristische Typisierung der Motive und ihre Austauschbarkeit mit gewissen Einschränkungen auch für die emblematischen Lebensbeschreibungen.

7. Motivübertragung von einem nichtjesuitischen Heiligen auf einen Jesuitenheiligen. Unter Girolamo Ruscellis Impresen über hervorragende Persönlichkeiten steht eine Komposition über Karl Borromäus. Das Bild zeigt einen von Schlangen bedeckten Hirsch, der auf einen Bach zurennt; das beigefügte Motto heißt: ,una salus“. Ruscelli erörtert in seiner Explikation die biblische Hirsch-Christus-Metapher, um dann von einem Psalmvers (Ps. 42 (41),2) ausgehend das Bild der klaren Quelle zu erläutern. Seiner Deutung nach

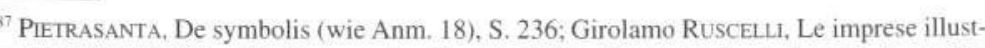
ri, Venetia 1584, S. 160-162. (Erstausgabe: Venetia 1566); TYPotrus, Symbola (wie Anm. 75), Tom. III, S. 127-129: Bovio, Rhetoricae suburbanum (wie Anm. 58), S. 30-31. Nr. III; Symbola in tractu (wie Anm. 49), f. bl/b.

${ }^{88}$ Pietrasanta, De symbolis (wie Anm. 18), zitiert bei Boschius, Symbolographia (wie Anm. 30), Cl. I. Nr. DXX: Imago Primi Saeculi (wie Anm. 24), S. 330; Bovio, Rhetoricae suburbanum (wie Anm. 58), S. 22-23, Nr. III. 
sehnte sich Karl Borromäus nach dem Wort Gottes, d.i. nach der klaren Quelle, wie es den Hirsch, der sich von den Schlangen befreien will, nach dem klaren Wasser des Baches dürstet.

Unter den Kardinal-Impresen des Typotius wird Karl Borromäus mit der gleichen Komposition bedacht. Der Kommentar ist ausführlicher als der bei Ruscelli, erwähnt diesen auch nicht, sondern beruft sich auf die Naturgeschichten von Oppian und Plinius und fügt hinzu, die Darstellung passe wegen seiner Tugenden, seines klaren Denkens und seiner Stärke auf den Kardinal. Pietrasanta benutzt dieselbe Komposition, um den 1610 heiliggesprochenen Karl Borromäus emblematisch zu vergegenwärtigen. Im Gegensatz zu Ruscelli und Typotius, die Hirsch und Quelle als gleichrangige Motive behandelten, stellt er in seiner kurzen Explikation die Quelle in den Vordergrund und interpretiert sie als das aus dem Quell des Heils geschöpfte Wasser (vgl. Jes. 12,3) ${ }^{89}$.

Etwas später sind die Motive bei einem anderen Jesuiten, bei Joannes Vincartius, teilweise umgedeutet geworden. Die Bildstruktur wurde vereinfacht, zugleich aber durch ein narratives Moment ergänzt, das sich auf eine Lebensstation des dargestellten Heiligen bezog. Aus dem Leben des Aloysius Gonzaga wählte Vincartius die Szene, als Aloysius den Brief des Generals Aquaviva über seine Aufnahme in den Orden erhält. Links sieht man die Aushändigung des Briefes, rechts den zur Quelle rennenden Hirsch. Im Mittelpunkt sowohl des neuen Mottos („Vivit, si reperrit undam“) als auch der Explikation in Versen steht, genauso wie bei Pietrasanta, die Quelle. Zum einen deutet Vincartius den Namen Aquavivas allegorisch (als aqua viva), zum anderen setzt er die Quelle mit dem Jesuitenorden gleich. Damit wurde das Hauptmotiv der auf Karl Borromäus verfaßten Impresa auf Aloysius Gonzaga übertragen ${ }^{90}$.

Das gleiche Motiv findet man, wenngleich nicht auf Personen bezogen, unter anderem bei Camerarius, Covarrubias Orozco und Heinsius. Camerarius übernahm sogar das Motto von Ruscelli (,una salus“), erweiterte jedoch den ursprünglichen Sinn des Emblems und benutzte es als Ausdruck des „Rettung durch Gott“-Gedankens. Mit je einem anderen Motto (,,Occido et manduco“, vgl. ApGesch. 10,9 bzw. ,solatium, non auxilium“) steht das Motiv bei Covarrubias Orozco und bei Heinsius; bei dem ersten drückt es die

${ }^{89}$ RuscelLl, Le imprese (wie Anm. 87), S. 90-92; TYPotIus, Symbola (wie Anm. 75), Tom II, S. 49-51: Pietrasanta, De symbolis (wie Anm. 18), S. 11.

${ }^{90}$ Vincartius, Sacrarum (wie Anm. 24), S. 164-168.
Idee der „Sündenvertilgung durch den Priester“, bei dem zweiten die der "Sehnsucht nach der Geliebten" aus ${ }^{91}$.

8. Das Motiv wird in seiner Anwendung auf einen Jesuitenheiligen durch einen spezifisch jesuitischen Gegenstand konkretisiert. Das der Naturwissenschaft entlehnte Motiv des Destillierkolbens war in der Emblematik des 16. Jahrhunderts beliebt, und im 17./18. Jahrhundert begegnet man ihm ebensooft. In Carlo Bovios Ignatius-Serie wird die Wahl des Heiligen zum General durch eine spezifische Form der stillatoria versinnbildlicht. Es handelt sich dabei um eine Konstruktion, die mit einem Ofen kombiniert, mit mehreren Ausgangsrohren ausgestattet und so zur gleichzeitigen Destillation verschiedener Stoffe geeignet war. Darum das Motto ,satis omnibus unus“, eins reicht für alle, nämlich ein Apparat genügt zur Reinigung von mehreren Stoffen. Zusammen mit der Inschrift, den Explikationen und dem Bild soll dadurch die Einstimmigkeit vergegenwärtigt werden, mit der die Ordensmitglieder Ignatius zum General gewählt haben ${ }^{92}$.

Dieser spezielle, Fornax Spagyrica genannte Destillierapparat war im Collegium Romanum in Betrieb. Seine Idee und Verwirklichung geht aller Wahrscheinlichkeit nach auf Athanasius Kircher zurück. Kircher hatte die Aktivität des Ätna mit der Fornax eines Destillierapparates verglichen ${ }^{93}$, und diese Beobachtung hat womöglich zum Bau der Fornax Spagyrica beigetragen. Der Apparat muß nach 1637-38 und vor 1655, dem Erscheinungsjahr des Ignatius-Emblems von Bovio, gebaut worden sein, seine früheste Beschreibung jedoch ist uns erst aus dem 1664 veröffentlichten Werk Kirchers bekannt.

Bei der emblematischen Darstellung von Papst Alexander VII. hat Bovio das Bild des Destillierapparates noch einmal verwendet. Außer bei ihm kommt die „Stillatoria jesuitica“ im Repertorium von Boschius vor. Die fünf mit Kupferstichen illustrierten Embleme, in denen sie dort figuriert, versinnbildlichen zusammen mit verschiedenen Motti den blutigen Schweiß Christi, das Gebet des Franz von Sales, die Reue, die geistige Arbeit und die Facultas

${ }^{91}$ HenKEL - SCHÖNE (wie Anm. 66), Sp. 470-471; vgl. Michael BATH, The Image of the Stag. Iconographic Themes in Western Art, Baden-Baden 1992, S. 289-297.

${ }^{92}$ HeNKEL - SCHÖNE, (wie Anm. 66), Sp. 1406-1407; FERRO DE' RotARI, Theatro (wie Anm. 79). S. 136; Abgetrocknete Thränen, Nürnberg-Frankfurt 1698. Sinnbild IV: Bovio. Ignatius insignium (wie Anm. 47), Nr. 58. 172-174.

${ }^{93}$ Athanasius KIRCHER, Mundus subterraneus, Tom. II, Amstelodami 1664, S. 392; Joscelyn GoDwIN, Athanasius Kircher, a Renaissance Man and the Quest for Lost Knowledge, London 1979, S. $84-85,92$. 
medica. Nach den Angaben von Boschius wurden diese Embleme in der Brüsseler Werkstatt der Jesuitenemblematik, weiterhin von zwei Jesuiten bzw. Exjesuiten, Menestrier und Emanuele Tesauro, verfertigt ${ }^{94}$.

\section{Zusammenfassung}

Die emblematischen Viten der Jesuitenheiligen wurden in enger Bindung an die Bedürfnisse und praktischen Zwecke des Ordens verfaßt, sie markieren eine bestimmte Periode in der Geschichte der geistlichen Emblematik. Die Gattung erlebte ihre Blüte um die Mitte und in der zweiten Hälfte des 17. Jahrhunderts, danach folgte ein rascher Verfall. Die letzte Kanonisation, die im Orden auch in der Form von emblematischen Viten gefeiert wurde, war die des Franciscus Regis. Neue Serien sind nach der Mitte des 18. Jahrhunderts nicht mehr enstanden, nur noch in den mittel- und osteuropäischen Ordensdruckereien wurden einige Neuausgaben publiziert. Einer der letzten unter den jesuitischen Theoretikern, der sich mit Emblematik befaßt hat, Ignatius Weitenauer, erwähnt die Heiligenembleme überhaupt nicht, und selbst die Möglichkeit, die Jesuitenemblematik gesondert zu behandeln, taucht bei ihm nicht auf. Seine Werke sind ein Zeichen dafür, daß die spirituelle Kraft der Emblemtradition erschöpft war und ihre Pflege im Orden endgültig abstarb ${ }^{95}$

Vom untersuchten Teilgebiet her beobachtet, ist ein „Paradigma jesuitischer Emblematik" gut zu erkennen. ${ }^{96}$ Die wichtigsten Merkmale der Jesuitenemblematik können thesenhaft in den folgenden Punkten zusammengefaßt werden.

1. Die Jesuiten pflegten die Heiligenemblematik nicht um ihrer selbst willen, sondern ordneten sie konsequent den Ordensinteressen, den Kanonisations- und Kultverbreitungsbestrebungen unter ${ }^{97}$. In der Entstehung und

${ }^{94}$ Bovio, Rhetoricae suburbanum (wie Anm. 58), S. 62-63, Nr. IV; Boschius, Symbolographia (wie Anm. 30), Cl. I. Nr. LV, Nr. CCCXXXVIII, Nr. DCXCV. Cl. III. Nr. DXVIII, Nr. DCLIII.

${ }^{95}$ Ignatius WeITENAUER, Symbolica, epigrammata, lapidaria libri III, Augustae Vindelicorum-Friburgi 1757: DERS.. De modo legendi et excerpendi libri II. Augustae Vindelicorum 1775 , S. 579-584

${ }^{96} \mathrm{G}$. Richard DIMLER, The bee-topos in the Jesuit emblem book: themes and contrast, in: Alison Adams - Anthony J. Harper (eds.). The Emblem in Renaissance and Baroque Europe: Tradition and Variety. Selected Papers of the Glasgow International Emblem Conference, 13-17. August, 1990, Leiden-New York-Köln 1992, S. 229-246, hier: S. 238.

"7 BremenenBCH, Der Emblematiker (wie Anm. 3), S. 70-78.
Verbreitung der Heiligenembleme spielten der Gelegenheitscharakter, die Emblemwerkstätten des Ordens, die Jesuitenautoren und die im Auftrag des Ordens tätigen Druckereien und Verlage eine bestimmende Rolle.

2. Bis zur Mitte des 18. Jahrhunderts entsprechen die emblematischen Heiligenviten im wesentlichen den Regeln und Forderungen der jesuitischen Emblemtheorie. Auf eine Praxis, die von den durch die Theoretiker festgelegten Vorstellungen abgewichen wäre, weisen die Drucke nicht hin. Theorie und Praxis der Jesuitenemblematik sind ohne die Bildtheorie, deren zentrales Element die ars memorativa bildet, überhaupt nicht oder nur schwer zu interpretieren ${ }^{98}$.

3. Die Jesuitenemblematik als Lehrmethode war vom verstärkten Bestreben geprägt, die Embleme allgemein verständlich und möglichst erkennbar zu gestalten sowie die esoterischen Züge weitgehend zurückzudrängen.

4. Infolge der Forderung nach claritas wurde die eindeutig gemachte similitudo in den Mittelpunkt gestellt. Zur Geltung kam ein System der Anforderungen, das von der "similitudo idonea“ geleitet war (dignitas, funktional festgelegtes Motivarsenal, Ausschließlichkeit der positiven Beispiele).

5. Der dreiteilige Aufbau imago-lemma-explicatio wird infolge der praktischen utilitas, des pädagogischen Zwecks, erweitert. Die vom Motto getrennte Inschrift in Prosa, die den Leser bereits im voraus über den Gegenstand der dargestellten Szene aufklärt, wird zur Regel. Die Multiplizierung der Explikation in verschiedenen Formen (Prosa, Elegie, Ode usw.) wird ebenfalls zur Praxis.

6. Anhand des Heiligenemblems der Jesuiten läßt sich der Prozeß verfolgen, in dessen Verlauf aus der Vita bzw. aus einzelnen Lebensstationen durch allegorisch-symbolische Transformation ein abstrakter Ausdruck entstand. Neben dem Bildmotiv steht das Motto nicht als etwas Gleichwertiges, es dient nur zur Vertiefung des im Bild versteckten symbolischen Bedeutungsinhaltes und zur Erleichterung des Verständnisses.

7. Die Explikationen (Kommentare) mit ihren verschiedenen Formen in Versen oder in Prosa liefern uns ein Bild des religiösen und humanistischen Wissensgutes, das in den Jesuitenschulen vermittelt wurde. Zudem

${ }^{98}$ Wolfgang Neuber, Imago und Pictura. Zur Topik des Sinnbilds im Spannungsfeld von Ars Memorativa und Emblematik (am Paradigma des .Indianers"), in: Wolfgang Harms ( $\mathrm{Hg}$.), Text und Bild, Bild und Text. DFG-Symposion 1988, Stuttgart 1990, S. 245-261, dazu die kritische Diskussion S. 309-314. 
spiegeln sie die konsequente Anwendung der poetischen und rhetorischen Regeln, die Wirkung der in den Exercitia spiritualia enthaltenen Theologie sowie die spezifisch jesuitischen Zielsetzungen wider.

8. Ein grundlegend neues Embleminventar haben die Jesuiten nicht geschaffen, in erster Linie schöpften sie aus der gemeinsamen Emblemtradition. Die Texte und Bilder lassen sich zu einem weit höheren Anteil mit der Tradition der weltlichen Emblematik in Zusammenhang bringen, als das bis jetzt angenommen wurde ${ }^{99}$. Die humanistischen Embleme wurden deformiert, ihre Motive in assoziativer und kompilativer Weise verknüpft, die ursprünglichen Bild- und Bedeutungsstrukturen wurden aufgelöst und verschmolzen. Es bildeten sich Übergangsformen zwischen der allegorischen Bildschöpfung und dem Emblem heraus.

9. Im Hinblick auf das Verhältnis von „,utile et dolce "läßt sich feststellen, daß die delectatio nicht das allerwichtigste Ziel darstellt ${ }^{100}$. Die Jesuiten verstanden sie in erster Linie als ein untergeordnetes Mittel, um die religiösen und moralischen Ideen des Christentums zu illustrieren, wirksam zu vermitteln und im Gedächtnis einzuprägen.

10. Im Verhältnis zwischen Bild und Text verschiebt sich die Betonung auf den Text. Statt der künstlerischen Darstellung tritt die rationale und emotionale Wirkung in den Vordergrund.

11. Die Besonderheit des jesuitischen Emblems ergibt sich aus der Wahl der Motive, aus ihrer Zusammenfügung und der Bestimmung des Kontextes, in dem die emblematischen Kompositionen stehen sollen.

12. Eine besondere Eigentümlichkeit besteht in der Bestrebung, eine möglichst große Popularität, eine Art Gesamt-Spektakel zu verwirklichen. Ein Vergleich des vorgeführten Materials mit emblematischen Viten nichtjesuitischer Heiligen und mit den entsprechenden Zeugnissen der außerliterarischen Emblematik ist wünschenswert.

\section{ఏ( 6 ) 絁}

\section{Ab ip o primo rationis ufu folide profert monumenta virtutis.}

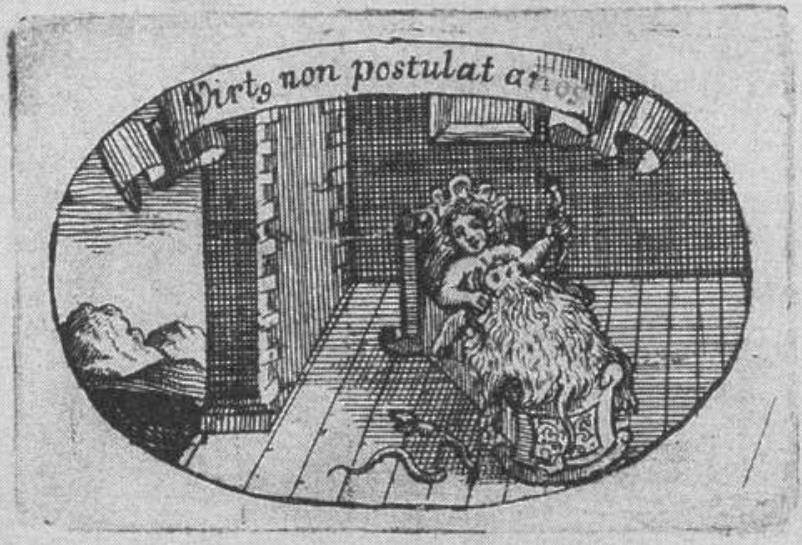

PArvulus Alcides binos eliferat angues; Alveus, infantum ftrata, palæftra fuit.

Quòd virtus generofa graves non poftulet annos,

In puero, fenij gloria, Koftka probat.

Non juvat ætatis Pyliæ numerare fenectam: Námque vir in puero eft, in fene fape

puer.

${ }^{99}$ G. Richard DimLER, A bibliographical survey of Jesuit emblem authors in German-speaking territories. Topography and themes, in: Archivum Historicum Societatis Iesu 45 (1976), S. 129-138; DERS.. The Imago Primi Saeculi (wie Anm. 3), S. 433-448.

${ }^{100}$ DiMLER, The Imago Primi Saeculi (wie Anm. 3), S. 446

I Antonins Mauisperg, Vita divi Stanislai Kostkae, Viennae (1726), S. 6: „Virtus non postulat annos", Kupferstich, $43 \times 52 \mathrm{~mm}$ 


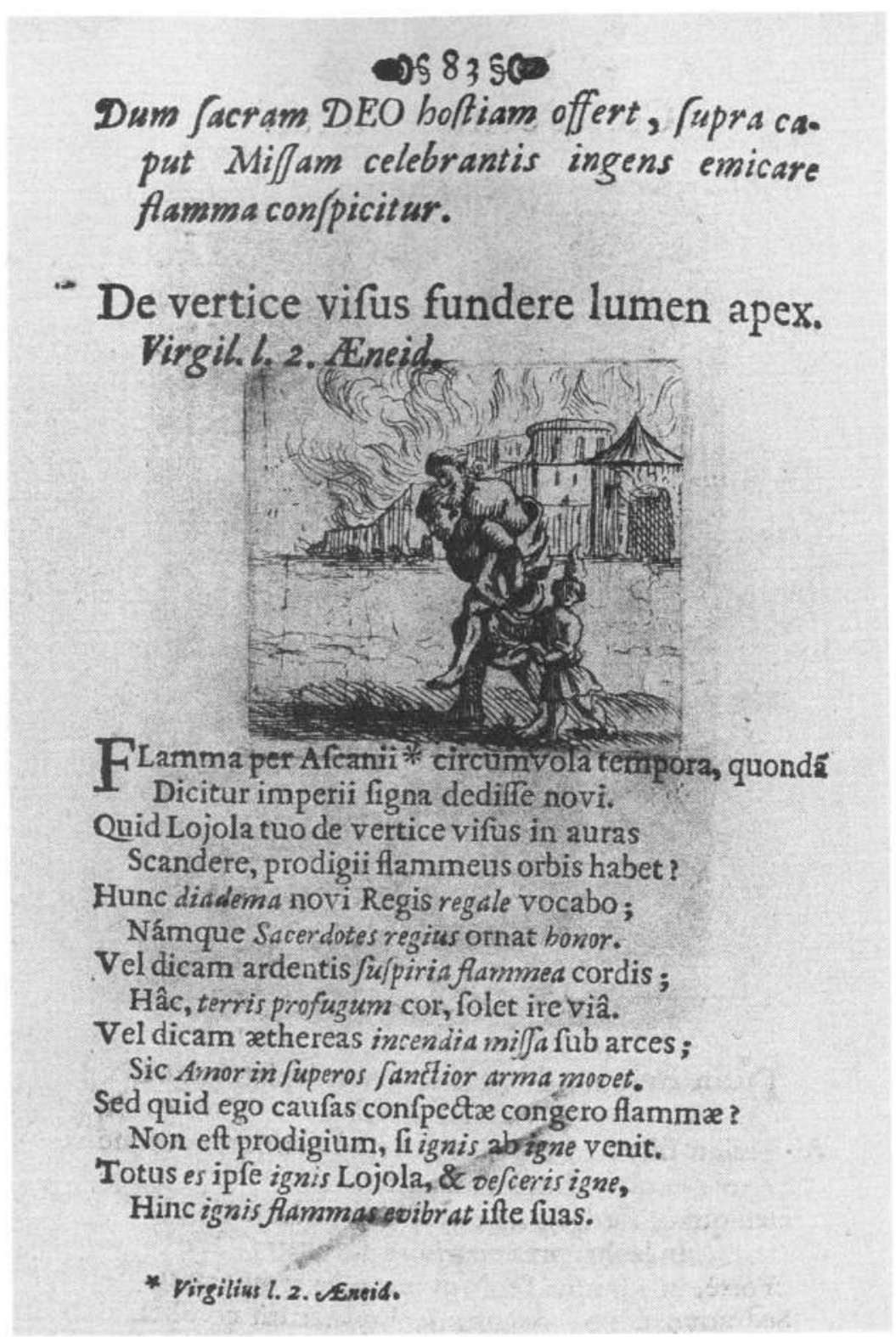

2 Ignatus Querck. Acta S. Ignatii de Lojola, Viennae 1698, S. 83; „De vertice visus fundere lumen apex. Virgil. L. 2. Aeneid, “, Kupferstich, $36 \times 41 \mathrm{~mm}$

\section{Sacrarvm HeroidVM}

\section{Xauerias Iaffo Parenti.}

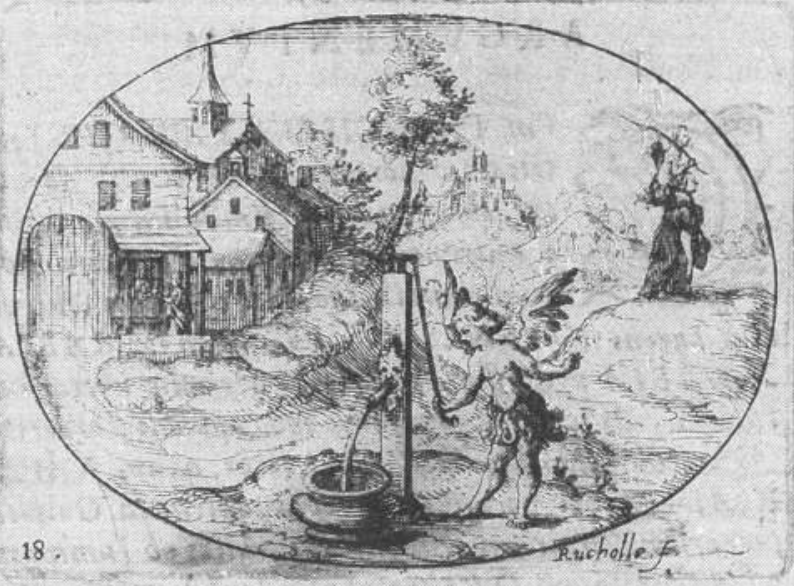

\section{$S \Upsilon M B O L V M$.}

Vt antlix aqua, eò largiùs confluit, quò effunditur: fic \& liberalitas Iaffi $\mathrm{Pa}_{\text {a }}$ rentis in Francifcum filiuin, olim familix, atque orbi ingentem fructum datura eft: fiquidem $a b$ his Xasuerij ftudijs falus laponum, Indorumaque pendebat. Iure igi tur hie apporas quod, antlix: Semper maior erit, quanizim fe ejjscderit vnda.

\section{Xauerias}

3 Joannes Vincartius, Sacrarum heroidum epistolae, Tornaci 1640, S. 132: „Semper maior erit, quantum se effuderit unda", Kupferstich, P. Rucholle, $45 \times 68 \mathrm{~mm}$ 
288.

IN S I G NE LXXV. OBSTINATYM IVDAEVM AD CHRISTIANAM FIDEM CONVERTIT TRIBVS HIS NON AMPLIVS VERBIS, MANE, NOBISCVM ISAAC,

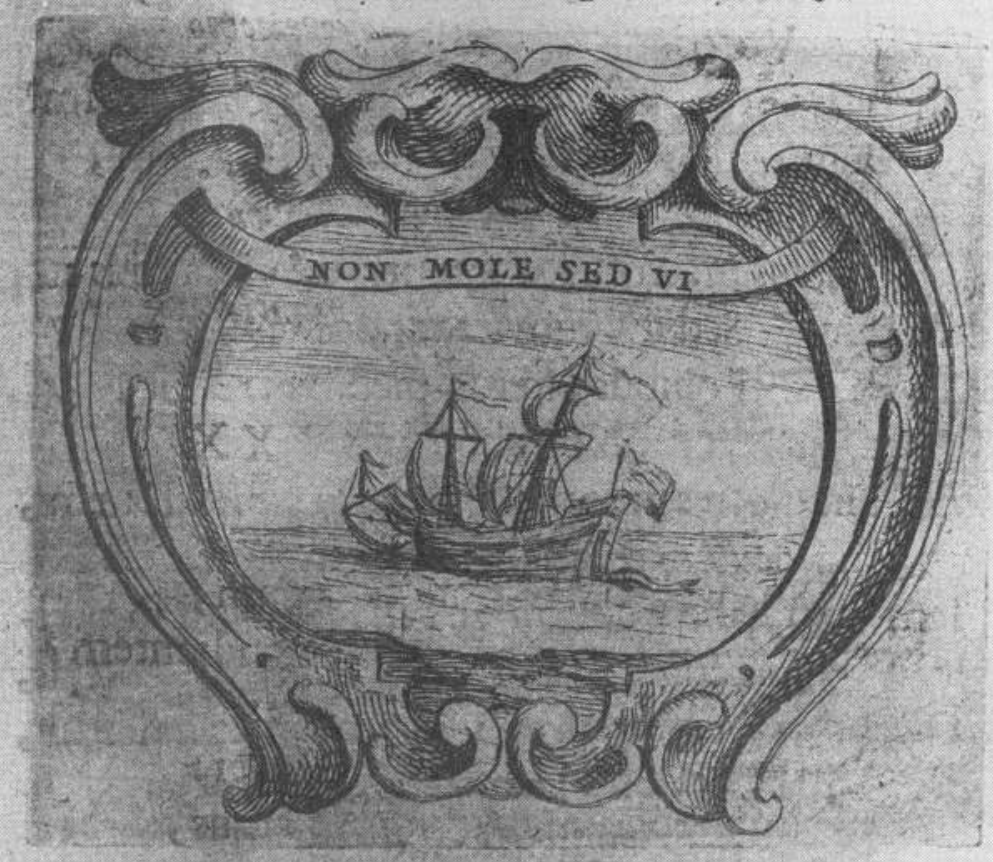

Affixa naui remora, pifcis tantulus, illam, ne moueri loco poffit tenet NON MOLE,SED VI. Qui obftinatum Iudai animum tribus non amplius verbis mutare potuit, quantam in ijs vim habebar Ignatius :

\section{EPI-}

4 Carlo Bovio, Ignatius insignium, Romae 1655, S. 238: .. Non mole sed vi“, Kupferstich, G. Castellus, $77 \times 89 \mathrm{~mm}$ $\infty 1005$

In Sançtorum numerum ì Gregorio $X V . \Phi_{\text {ant. }}$ Max.adjcribitur I2.Martii An, $1 \sigma_{22}$.

Coleftibus intulit aftris, Ovid. Metam.

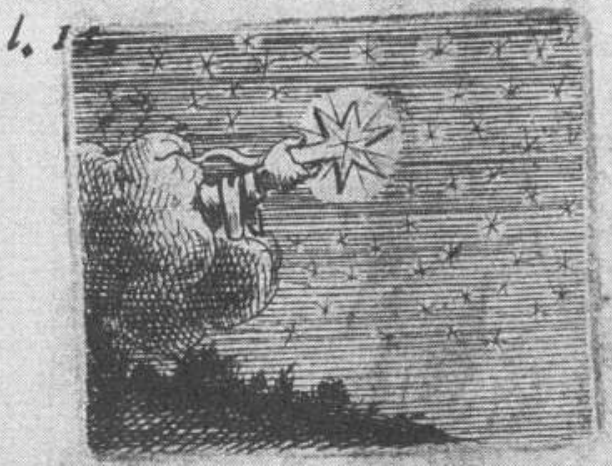

Gnis es, ignis eras, \& in omne IGNATIUS ævvum

Ignis eris; nomen fic ait, acta probant.

Hinc te Gregorius coeleftibus intulit aftris; Perpetuum tellus lumen habere nequit:

Ifta Deum folum docrit Deus ipfe decere;

Lampadées ejus enim, lampades ignis erant.

Gratulor IGNATI translatum nomen ad aftra; Qud vifus dudum f piritus ire tous.

Inde tibi poteris pofita illuftrare trophæa, Inde tenebrarum Principis aufa premes.

Inde vides, quali igne flagret domus utraque Phrbi, Inde tuis addes in nova bufta faces.

Inde mihi minimam, $\mathrm{O}$ ! ignis ! concede favillam,

Ardebo, qui nunc frigeo carbo tuus.

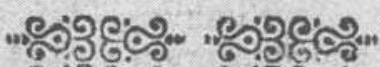

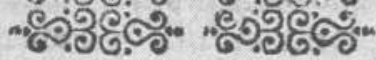

5 Ignatius Querck, Acta S. Ignatii de Lojola, Viennae 1698, S. 100: „Coelestibus intulit astris. Ovid. Metam. 1. 15, “, Kupferstich, $38 \times 43 \mathrm{~mm}$ 
LIBER QVINTVS. SOCIETAS HONORATA, 72I

Xauerius, orbe peragrato, moritur in littore Chinarum.

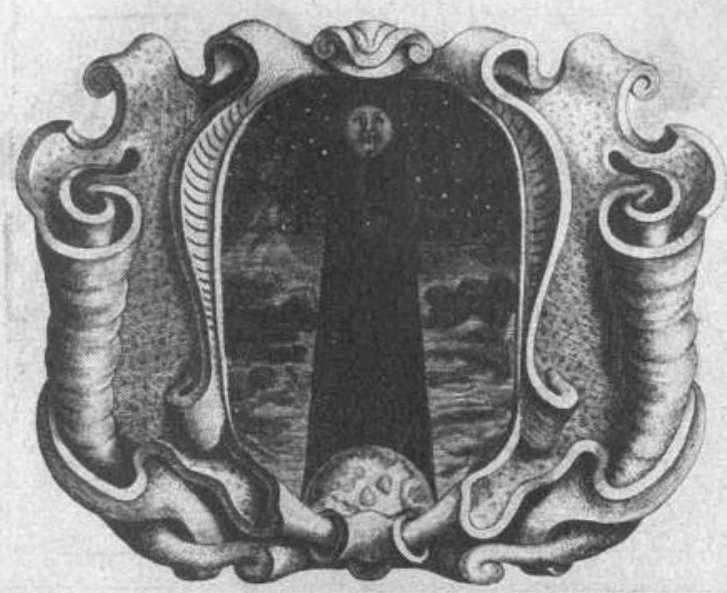

Tum te terra teget, cùmtotum impleueris orbem. Ingite fronde caput: Xaweriut or be Laboris
Deficit impleto: cingite fronde caput. Timplius ille mibil portuit fuperaddere capis. Tota peragrata ell India, iam fatis eff. Chine, Goa, lapon, Ternata, Moluca, tot vrbes, Que toties inite cauffa fuire ris;

Totáue alia gentes, sot primo Jubdita Pbabo

Littora, Judoris plus babiere fatis.

Ergo vel extreman cùm nil Jupereffet ad Eon; Ipfis terrarum finibus immoritur.

Immoritur cosis ingentibus, orbe replete:

Cynebia fic etiam plena perire folet.

Cyntbia terrarum, celi Erancif cus ab rombra

Palluit: exanomis viultus, amantris erat.

Obruatur tenebris of furuid Cynshia nube:

Francifcus radis obrut af ra fuis.

Con iubar hoc magice carmen senuauerit artis:

Nil opus ad numeros ara repulfa fonent:

Perfat, \& integros vbi vits reliquerit artur,

Mausus ab extinito corpore bumen erit.

$$
\text { YyYy }
$$

B. Fran-

6 Imago Primi Saeculi, Antverpiae I640, S. 721: „Tum te terra teget, cum totum impleveris orbem", Kupferstich, $112 \times 133 \mathrm{~mm}$

\section{E $R \quad S \quad I \quad L \quad I \quad A$ $\begin{array}{llllllllllllll} & C & O & R & T & E & S & E\end{array}$ D E M O N T I.}

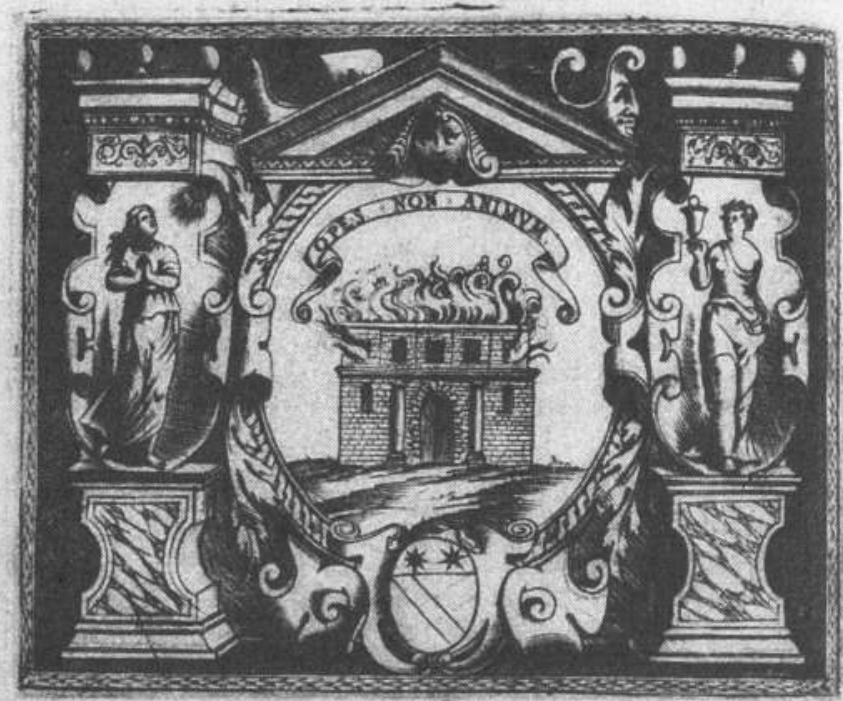

Poprors RA LE MOLTE BELLECAGIONI, per le quAe. 2 . 131203 prele fia ora, \& fia per effer di continuo in fommo pregio,

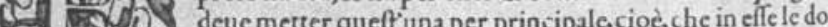
c) ( 40202

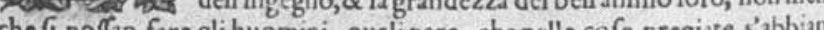
chefiporian are gli huomini, quallpare, che nelle cofe pregiate s'abbiano dometira nefcante ufurpata la maggioranza.Che per cler le doneper dinario di piu delicata, a gentil compleisinne, shanno gli huomini fartalor a gloria dell arme.Et per cier efsi parimente pia attialle fatiche, che fir ricercano ne gli Itudij, \& inlieme pru arditi, \& piu conueneuoli ad vfarle nei palazzi,nelle fcuole, nelle piazze, \& negli altri luoghi, oue per guadagno, \& per vfo de' publici, ò de' particolaris'adoprano, hanno efsi huomini anco in qnefto

7 Girolamo Ruscelli, Le imprese, Venetia 1584, S. 160: „Opes non animum", Kupferstich, Giacomo Francho, $85 \times 115 \mathrm{~mm}$ 


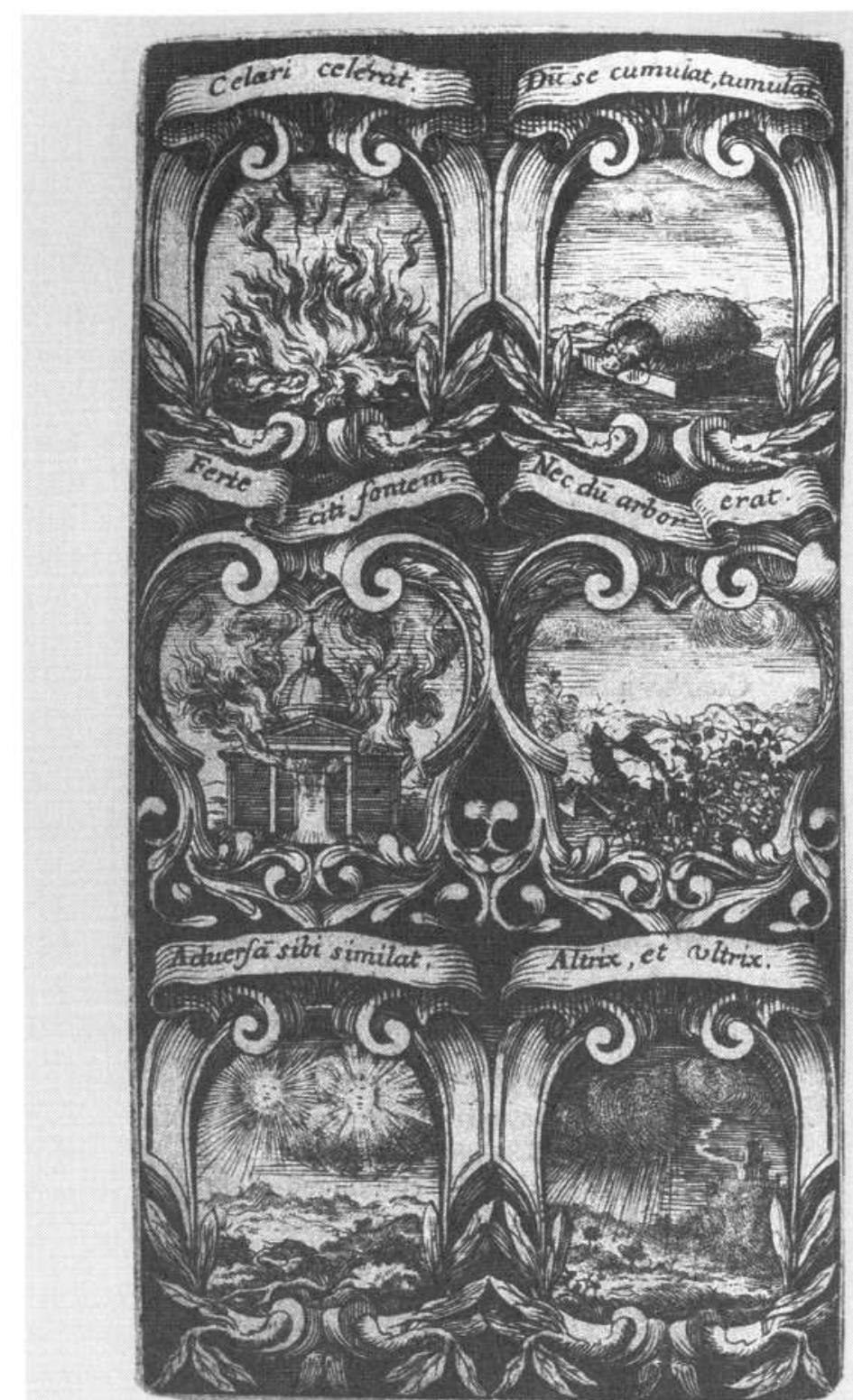

8 Carlo Bovio, Rhetoricae suburbanum. Romae 1676, S. 30-31, Nr. III: „Ferte citi fontem “. Kupferstich, $110 \times 61 \mathrm{~mm}$ (Plattengrôsse)
L I B E R P I M V S.

$\&$ Viro ab elegantiâ morum \& litterarum inprimis claro. II. verò eft PAvir Aresir,Clerici Regularis, \& qui,edito etiam volumine de Symbolis facris, Italico idiomate, adeptus eft eruditionis famam.

Sed enim, vt in medium adducam exDrvis ipfis aliquem, qui fuum fibi Symbolum concinnauerit,

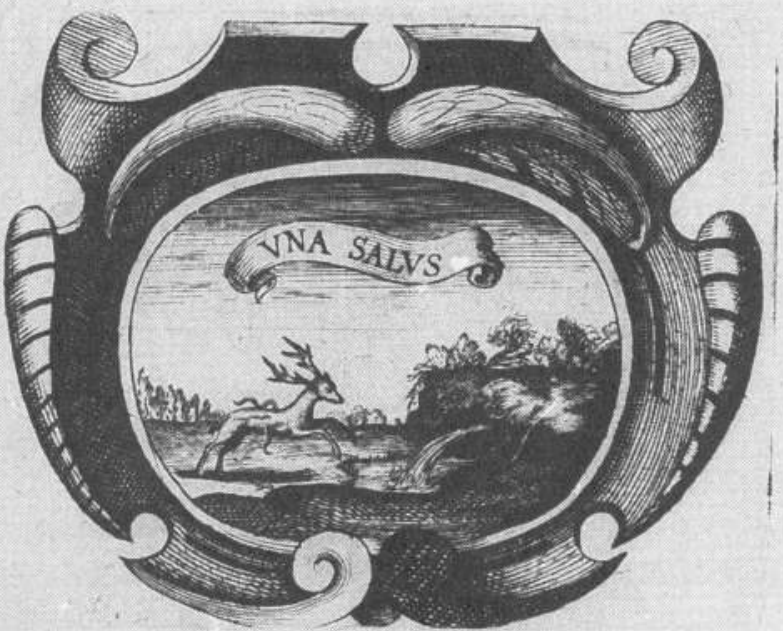

S. Carolvs Borromaevs Mediolani Archipreful, \& S.R.E. Cardinalis, Ceruo vfus eft, qui aduerfus anguium venena fontem quxrit, cum hac Epigraphe: VNA salvs. Solitus videlicet fibi vnicè in aduerfis mederi, hauriendo aquas de fontibus Saluatoris.

B 2

CAPVT

9 Silvestro Pietrasanta, De symbolis heroicis libri IX. Antverpiae 1634, S. 11: „Una salus“ Kupferstich, $68 \times 85 \mathrm{~mm}$ 
$1 \% 2$

IN I I N E LVIII

\section{SOCIETATIS IESV PRAEPOSITVS, GENERALIS} ELIGITVR.

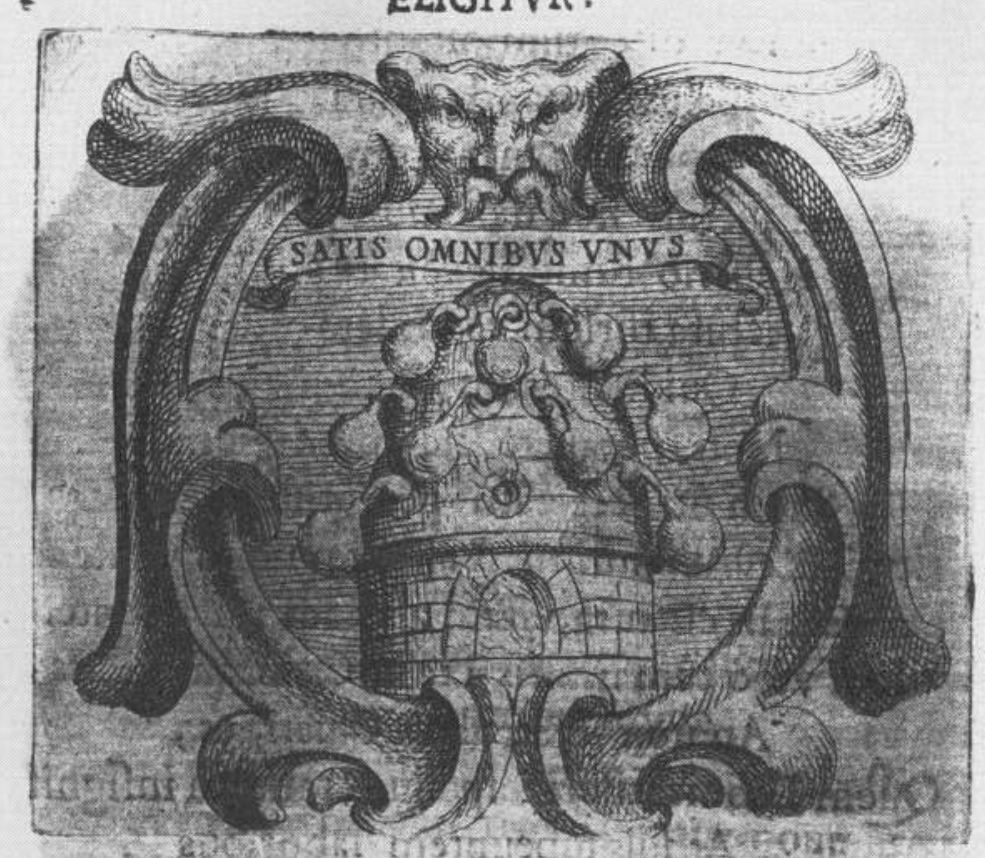

In ftillatoria machinz formacula mediys ignis inclufus diffo fris circum vituis SATHSOMNIZVS VNVS oft. No diftillatrs fudoribusjpir fe in talifem eliquen proxim orum, ab pno lgnatij igne, qurm fuprtmo Magilleataiadmotumfibi nediam (tacuere, Socij on aes caloré accipiunt.

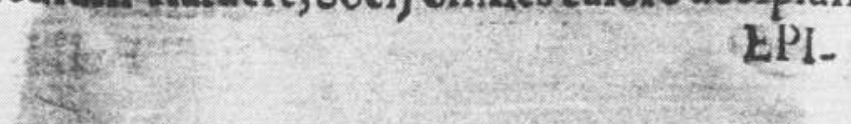

10 Carlo Bovio, Ignatius insignium, Romae 1655, S. 172; „Satis omnibus unus", G. Castellus, $75 \times 87 \mathrm{~mm}$

\section{Exzeptionelle Weiblichkeit und gestörte Ordnung}

Zur Kontinuität literarischer Entwürfe der sinnlichen Frau

von Karina Kellermann und Renate Stauf

Die Geschichte der ,imaginierten Weiblichkeit“1 ist zugleich eine Problemgeschichte menschlicher Sinnlichkeit. Als tiefverwurzelte Angst vor einem unkontrollierbaren, der Vernunft entzogenen Triebschicksal offenbart die Emanzipationsgeschichte des menschlichen Geistes ihr Janusgesicht vor allem in jenen sinnlichen, betörenden, unheil- oder sogar todbringenden Frauen, die seit jeher Literatur und Kunst, Geschichte und Mythologie bevölkern. Daß sie nicht Bilder real existierender Weiblichkeit sind, sondern überwiegend Männerphantasien entsprechen, ist spätestens seit dem Aufkommen der feministischen Literatur- und Kunstkritik zur opinio communis geworden. Allerdings hat die Diskussion der letzten Jahre auch gezeigt, daß durch die allzu enge Begrenzung auf die misogynen Aspekte derartiger Weiblichkeitsentwürfe der Blick für ihre Funktion innerhalb umfassender Versuche des gesellschaftlichen und literarischen Ordnungsstiftens verstellt wurde. So ist der epochenübergreifende Zusammenhang zwischen der Konzeption sinnlicher Frauen und der Gefährdung oder Störung von jeweils sehr spezifischen Ordnungssystemen bisher kaum untersucht worden. Dabei ist nicht zu übersehen, daß der Darstellung weiblicher Sinnlichkeit ein besonderes Gewicht zukommt, wenn gesellschaftlich etablierte Ordnungen - seien sie sittlichmoralischer, ästhetischer oder politisch-staatlicher Natur - in die Diskussion kommen. Als literarische Imagination wird das Weibliche offenbar gebraucht, um bestehende Ordnungen zu stützen oder neu zu bestätigen, gegebenenfalls neue Ordnungen zu begründen oder um jeglichen Versuch des Ordnungsstiftens fundamental in Frage zu stellen. Sieht man von den epochenspezifischen Varianten einmal ab, kristallisieren sich in den Gestaltun-

\footnotetext{
' Den Begriff programmatisch eingesetzt hat Silvia Bovenschen (1979).
} 